bioRxiv preprint doi: https://doi.org/10.1101/2021.08.17.456688; this version posted August 17, 2021. The copyright holder for this preprint (which was not certified by peer review) is the author/funder, who has granted bioRxiv a license to display the preprint in perpetuity. It is made available under aCC-BY-NC-ND 4.0 International license.

\title{
Decoupling the ATP-binding Domain in the CheA Kinase by Increasing Linker Flexibility Dramatically Alters Kinase Activity
}

\author{
Zachary Maschmann ${ }^{a}$, Siddarth Chandrasekaran ${ }^{a b}$, and Brian R. Crane ${ }^{a b *}$
}

${ }^{\text {a }}$ Department of Chemistry and Chemical Biology, Cornell University, Ithaca, NY 14850

${ }^{\mathrm{b}}$ National Biomedical Center for Advanced ESR Technologies, Cornell University, Ithaca NY 1485 ${ }^{*}$ For Correspondence: bc69@cornell.edu

Competing interests: The authors declare that no competing interests exist.

\begin{abstract}
In bacterial chemotaxis chemoreceptors regulate the cytosolic dimeric histidine kinase CheA. To test the role that interdomain linkers play in CheA regulation the linkers that connect the $\mathrm{P} 4$ kinase domain to the P3 dimerization domain (L3) and the P5 regulatory domain (L4) were extended and altered in variants of Thermotoga maritima $(\mathrm{Tm})$ CheA. Flexible extensions of the L3 and L4 linkers in CheA-LV1 (linker variant 1) allow for a well-folded kinase domain that retains WT-like binding affinities for nucleotide and normal interactions with the receptor-coupling protein CheW. However, CheA-LV1 autophosphorylation activity registers $\sim 50$-fold lower compared to wild-type. Formation of the CheA-LV1 / CheA WT heterodimer fails to rescue CheA-LV1 autophosphorylation and instead reduces the activity of the WT subunit. Neither CheA WT nor CheA-LV1 can phosphorylate P1 in a CheA dimer that contains a single P4 domain. Rescue of autophosphorylation activity in variants with a poly-alanine L3 or an L3 that maintains a heptad repeat suggest that positioning and conformational transitions of P4 depend on L3 assuming helical structure. Pulse dipolar ESR measurements indicate that the CheA-LV1 P4 domains are in close proximity whereas broader distributions in other variants correlate with increased activity. CheA-LV1 has a substantially larger hydrodynamic radius than does CheA WT by SAXS, despite the P4 domains assuming a closed, inhibited conformation. These results explain negative cooperativity in CheA nucleotide binding, demonstrate coupling between P4 disposition and P1 / P2 dynamics and underscore the importance of P4-P4 interactions and an L3 $\alpha$ helix in CheA activity and regulation.
\end{abstract}

\section{Introduction}

Chemotactic bacteria coordinate their motility to changing environmental conditions by sensing specific chemicals through a receptor-mediated process ${ }^{1-4}$. Increasingly, chemotaxis is being recognized as a key determinant of pathogenicity in motile bacteria and serves as a paradigm for 
signal transduction systems in general ${ }^{5-10}$. A highly conserved supramolecular protein infrastructure transduces chemoreception signals to regulate the motility engines of the cell ${ }^{3}$. Dimeric

transmembrane chemoreceptors, also known as methyl-accepting chemotaxis proteins (MCPs), bind attractants and repellants in the periplasmic space and undergo conformational shifts that cross the cell membrane and reach the cytosolically distal end of the receptor dimer ${ }^{3,4,10,11}$. Signal gain and sensitivity to minute changes in ligand occupancy permit chemotactic bacteria to track chemical gradients. The core chemosensory components form extended hexagonal arrays consisting of trimers of receptor dimers (TODs), a dedicated dimeric histidine kinase CheA, and an adaptor protein $\mathrm{CheW}^{2,11,12}$. The array superstructure facilitates cooperative responses and signal gain. CheR, a methyltransferase, and CheB, a methylesterase, modify specific MCP residues as part of an adaptational response to aid chemical gradient tracking over time 3,4,11,13-16. Receptor conformational signals modulate CheA autophosphorylation and therefore subsequent phosphoryl-transfer to the response regulator CheY. Phosphorylated CheY (CheY-P) binds to the flagellar rotor and directly affects flagellar rotation ${ }^{12}$. The molecular details of CheA autophosphorylation are therefore of central importance to understanding signal processing and transduction in chemotaxis. The best characterized chemotaxis system is that of $E$ coli, and it has long served as a model system for understanding bacterial chemotaxis in general. However, the thermostable chemotaxis proteins from the thermophile Thermotoga maritima $(\mathrm{Tm})$ have also been invaluable tools for structural and biochemical characterization of the chemotaxis proteins and their assemblies ${ }^{4}$.

CheA is comprised of five domains: a P1 substrate domain, a P2 docking domain, a P3 dimerization domain, a P4 kinase domain, and a P5 regulatory domain homologous to CheW. The P1-to-P2 and P2-to-P3 linkers (hereafter referred to as L1 and L2, respectively) are long and flexible (72 and 38 amino acids, respectively for Tm CheA), whereas the P3-to-P4 and P4-to-P5 linkers (hereafter referred to as $L 3$ and $L 4$, respectively) are short (2-3 amino acids each) $)^{17-20}$. The catalytic cycle of CheA involves P4 binding to ATP, subsequent binding of P4 to the opposite P1, transfer of the $\mathrm{Y}$ phosphate to a conserved histidine residue on $\mathrm{P} 1$ of the adjacent subunit, and P1 release ${ }^{17,21-26}$. ADP then exchanges for ATP in the P4 active site and CheY catalyzes phosphotransfer from P1 to a conserved CheY aspartate residue. Within the arrays, the P5 domain of CheA and its paralog CheW form rings with two types of interfaces that assemble the signaling units into extended hexagonal arrangements (Figure 1A) ${ }^{13,15,16}$. Conformational signals affecting CheA are propagated vertically along receptors and laterally throughout the arrays.

$E c$ CheA autophosphorylation depends on complexation with receptors and their ligand occupancy. Ec CheA has an "on / open" state when associated with chemoreceptors and an "off / sequestered" 
state when receptors are bound to chemoattractants. The free Ec CheA state is termed "closed". Tm CheA has two autophosphorylation levels: a receptor-complexed "sequestered" conformation that constrains the P1 domains and an "open" conformation that enables P1-CheY interaction (Figure 1B $)^{4,27}$. P1 phosphorylation decreases dramatically upon receptor inhibition ${ }^{28}$. Due to the long and flexible L1 and L2 linkers, the release of constrained P1 from the kinase core domains P3-P5 increases the hydrodynamic radius of the CheA dimer considerably, as evidenced by SAXS measurements ${ }^{29,30}$. The molecular details of the kinase conformations responsible for different autophosphorylation levels are not fully characterized. The isolated P4 domain phosphorylates free P1 domain with very low activity compared to full-length $\mathrm{CheA}^{4,29}$. However, for Tm CheA, the P3 and P4 domains increase kinase activity above CheA FL and well above P4 alone. Addition of the P5 domain, by contrast, is inhibitory. The ability of dimerization (conferred by P3) to dramatically increase activity suggests that either the L3 connection affects P4 catalytical activity or the proximity of the P4 domains in a CheA dimer does $\mathrm{so}^{4,29}$.

The CheW / P5 interface is critical to the control of kinase activity in vivo and perturbation of the interface has been shown to produce defects in CheA regulation by receptors ${ }^{13,31}$. It is increasingly thought that CheW transmits conformational signals to the kinase preferentially over the P5 domain and inhibition of CheA may involve exclusion of P1 from or sequestration of the P4 ATP binding site ${ }^{4,13,32}$. Indications that the P4, P1, and P2 domains are closely associated in the inhibited state of Tm CheA complicate the task of discriminating structural features responsible for kinase activation and inhibition ${ }^{30,33-35}$.

Changes to the L3 and L4 linkers have been demonstrated to greatly affect P4 kinase activity ${ }^{36,37}$. Point mutations of the L3 residues modestly reduce the basal autophosphorylation level of CheA, but also impair kinase activation by receptors ${ }^{36,37}$. Residue substitutions in the L4 linker, in contrast, both decrease and increase the basal autophosphorylation level of CheA even in the absence of the P5 domain and also impair the ability of chemoreceptors to modulate CheA activity ${ }^{31,36}$. Interestingly, recent results from cryo-electron microscopy experiments coupled with molecular dynamics simulations suggest that the position, dynamics, and hydrogen-bonding network of the P4 domain may be coupled to a secondary structure change in the L3 linker ${ }^{38}$. Additionally, distinct conformations of the P4 domains in "dipped" and "undipped" conformations accessible to CheA support the notion that regulation of P4 mobility is important in the CheA catalytic cycle ${ }^{16,38}$. Observations that increased proximity between the P4 domains is associated with the inhibited state of the kinase in pulse dipolar ESR (DEER) experiments corroborate this hypothesis ${ }^{30}$. In addition, strong negative cooperativity in nucleotide binding between the subunits of dimeric CheA further 
suggests that the P4 domains influence one another, either through direct contact or through conformational propagation, involving perhaps the P3 domains. Restriction of P4 movements by the L3 and L4 linkers, therefore, may play a further role in regulating kinase activity ${ }^{39}$.

To investigate the role of the L3 and L4 linkers on CheA activity, we have produced and characterized a series of $T m$ CheA variants wherein the L3 and L4 linkers are altered and extended to increase flexibility, alter P4 juxtaposition and favor helical conformational states (Figure 1C). These modifications serve to disconnect the P4 domain from the P3 and P5 domains and enforce alternate physical linkages. The variants interact with $\mathrm{CheW}$ and receptor domains and maintain proper folding of their P4 domains, which bind ATP and P1. However, the linker variants (LV) have altered P1 and P2 conformational dynamics, P4 positioning, autophosphorylation activity and temperature dependence of that activity. The most severely affected variant CheA-LV1 has very low kinase activity while displaying a close autoinhibitory association between its P4 domains. Extending the linkers further partially rescues activity, especially when the sequences favor a helical connection. The change in relative activity of the variants with temperature indicates that P4 movement may be important to complete the catalytical cycle. Subunit exchange coupled with phosphorylation assays, SAXS, cross-linking experiments and temperature dependencies of autophosphorylation imply that mobility of the P1 and/or P2 domains, the global dynamics of the kinase, linker flexibility, and basal level kinase activity are tightly coupled to conformational properties and interactions of the P4 domain. 
A

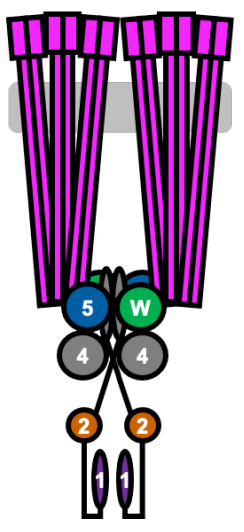

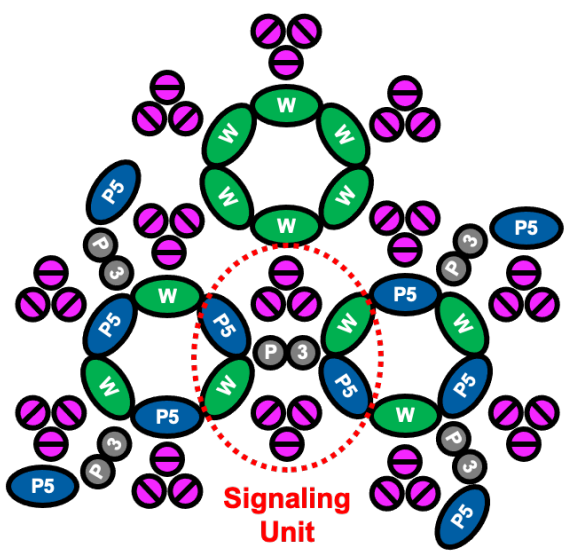

B
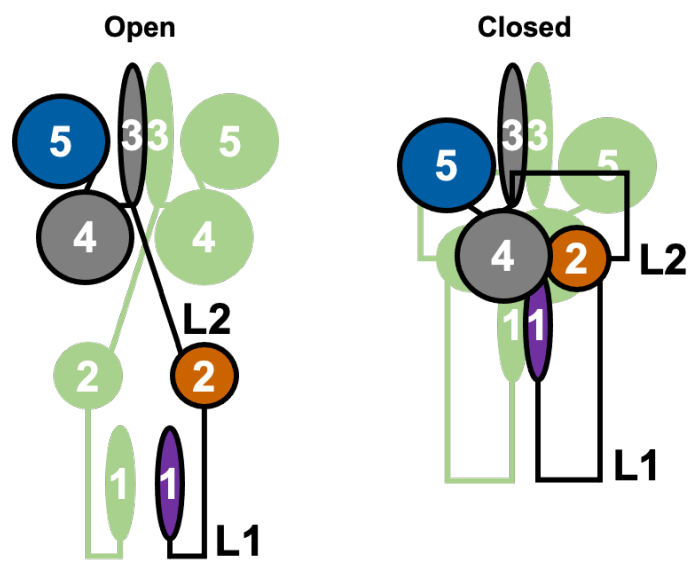

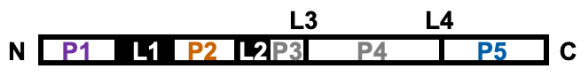

C

\begin{tabular}{l|l|l|}
\multicolumn{1}{c|}{ Linker 3 Sequence } & Linker 4 Sequence \\
\cline { 2 - 3 } CheA WT & P3-MKIRMVPISFVFN-P4 & P4-RLPLTLAI-P5 \\
CheA-LV1 & P3-MKIRGAGGSMVPISFVFN-P4 & P4-RLPLGGSGSTLAI-P5 \\
CheA-LV2 & P3-MKIRAAIAAIAMVPISFVFN-P4 & P4-RLPLGGSGSTLAI-P5 \\
CheA-LV3 & P3-MKIRGAGAGGSMVPISFVFN-P4 & P4-RLPLGGSGSTLAI-P5 \\
CheA-LV4 & P3-MKIAAAAAAAVFN-P4 & P4-RLPLTLAI-P5
\end{tabular}

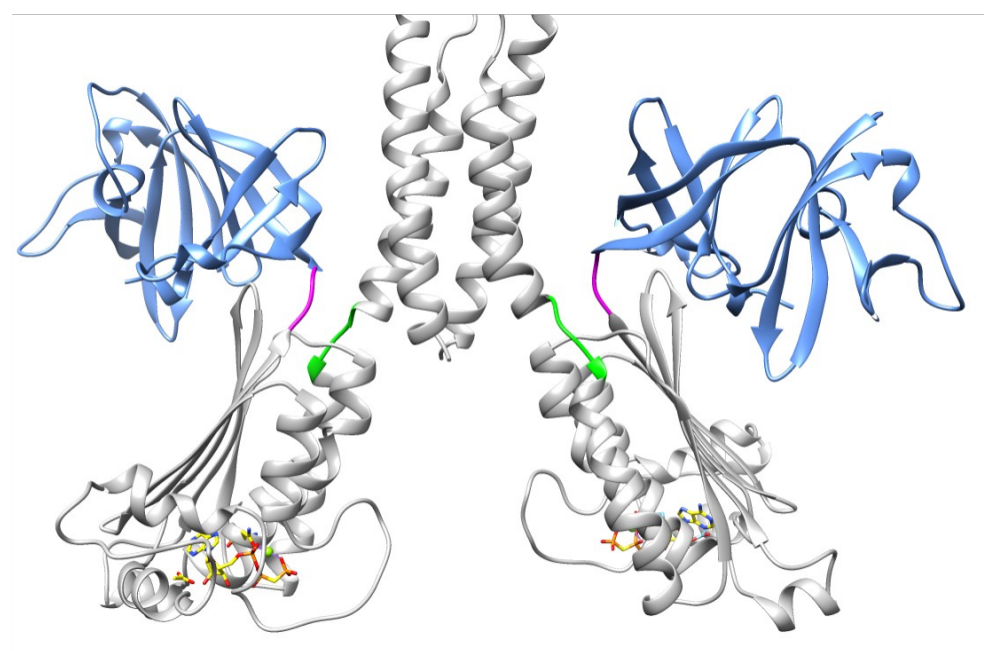

Figure 1 The multi-domain histidine kinase CheA is a central component of the receptor:kinase arrays that mediate chemotaxis. (A) The chemotaxis receptor:kinase array architecture. Left: Vertical view of transmembrane Trimers of MCP Receptor Dimers (TODs) that contact CheA and CheW (green) in the cytoplasm and regulate CheA autophosphorylation. Right: a lateral view of the extended arrays formed by neighboring signaling units. Two TODs, 2 CheW subunits, and a CheA dimer constituting a core signaling unit are circled in red. (B) The domain arrangements of $T$. maritima CheA WT in open and closed conformations. Schematic is based published crystal structures of domains and complexes (P1 from PDB ID: 1TQG; P2 from PDB ID: 1U0S; P3P4P5 from PDB ID: 1B3Q) and other biophysical data ${ }^{30}$. The $\mathrm{P} 1$ and $\mathrm{P} 2$ domains are connected by a long flexible linker (L1) and the P2 and P3 domains are connected by another long flexible linker (L2). 
Relatively short L3 and L4 linkers connect the P3 domain to the P4 domain and the P4 domain to the P5 domain, respectively. (C) Above: table showing linker modifications of CheA variants. The WT L3 and $L 4$ residues are colored green and blue, respectively. Residue insertions or changes are colored in black. Linker variants (LV) 1, 2, and 3 extend the L3 and L4 linkers. LV1 and LV3 are designed to maximize flexibility. Variant 2 is designed to produce a stable continuous $\alpha$-helix and promote association between the P4-P4 dimer interface. LV3 inserts a heptad repeat that would allow an inregister $\alpha$-helix but maintains flexibility. LV4 has a WT L4 length, but a poly-Ala sequence. Below: model of dimeric TmCheA P3P4P5 from PDB ID: IB3Q highlighting L3 and L4 (P3P4:gray, P5:blue, L3: light green, L4:purple, ADPCP shown as a stick model).

\section{Methods}

\section{Chemicals, Reagents, and Proteins}

All proteins used in the experiments presented in this study were derived from Thermotoga maritima and produced in E. coli. CheY (UniProt ID: Q56312), CheW (UniProt ID: Q56311), CheA WT (UniProt ID: Q56310), CheA P1-3 (residues 1-354), CheA-LV1, CheA-LV2, CheA-LV3, CheA-LV4, and Escherichia coli Tar $_{F O}$ Short were recombinantly expressed in BL21 E coli cells using a pet28a vector and purified by affinity chromatography as previously reported ${ }^{28}$. 2'(3')-O-(2,4,6Trinitrophenyl)-adenosine 5'-triphosphate (TNP-ATP) was purchased from Invitrogen as a trisodium salt and stored in the dark at $-20^{\circ} \mathrm{C}$ at $10 \mathrm{mg} / \mathrm{mL}$. DSSO was purchased from ThermoFisher. Copper (II) Sulfate and $(1,10)$-phenanthroline were purchased from VWR and subsidiaries. Tris, HEPES, $\mathrm{KCl}, \mathrm{NaCl}, \mathrm{MgCl}_{2}$ were purchased from Sigma. [Y- ${ }^{32} \mathrm{P}$ ]ATP was purchased from Perkin Elmer. ADP-NO was synthesized as previously reported ${ }^{40}$.

\section{CheA Autophosphorylation Assays}

CheA autophosphorylation was monitored by ${ }^{32} \mathrm{P}$ incorporation. All radioassays were carried out in $50 \mathrm{mM}$ MOPS pH 7.5, $150 \mathrm{mM} \mathrm{KCl}, 10 \mathrm{mM} \mathrm{MgCl}$. CheA WT and CheA LV samples were prepared at between 1.5-3 $\mu \mathrm{M}$ protein (consistent within each experiment) at a total volume of $23 \mu \mathrm{L}$. Following room temperature incubation for $\sim 15$ minutes, [Y-32P]ATP was added to a final concentration of $1 \mathrm{mM}$ and the reaction was quenched after 12 minutes using $4 x$ SDS-PAGE loading buffer containing $50 \mathrm{mM}$ EDTA pH 8.0. Elevated temperature experiments employed a heating block. The samples were loaded onto a 4-20\% Tris-glycine polyacrylamide protein gel purchased from Invitrogen. Gel electrophoresis was carried out for 35 minutes at $125 \mathrm{~V}$ constant voltage, and gels were dried in a Bio-Rad Gel Dryer overnight in cellophane. Exposure to the storage phosphor plate occurred for $>20$ hrs prior to imaging on a Typhoon Image Scanner. Gel bands were quantified using 
ImageJ. Some gels were rehydrated in water and stained using 10\% Acetic Acid, 25\% Ethanol in water containing $2.5 \mathrm{~g} / \mathrm{L}$ Coomassie Brilliant Blue, and subsequently destained with 10\% acetic acid and $25 \%$ ethanol.

\section{CheA Subunit Exchange and Disulfide Crosslinking}

CheA WT, CheA-LV1, and CheA P1-3 were reconstituted in $50 \mathrm{mM} \mathrm{MOPS} \mathrm{pH} \mathrm{7.5,} 150 \mathrm{mM} \mathrm{KCl,} 0.5$ $\mathrm{mM} \mathrm{MgCl}_{2}, 5 \%$ glycerol at $20 \mu \mathrm{M}$ and following either brief incubation at room temperature or a 5 minute incubation at $55^{\circ} \mathrm{C}$ to exchange subunits, $\mathrm{Cu}(\mathrm{II})$ and $(1,10)$-phenanthroline were added to final concentrations of 5 and $15 \mathrm{mM}$, respectively. The reactions were quenched with loading buffer after $8 \mathrm{hrs}$ of incubation at room temperature ${ }^{41}$. The resulting samples were subjected to gel electrophoresis on a 4-20\% Tris-glycine protein gel. The gels were stained in 10\% Acetic Acid, 25\% Ethanol in water containing $2.5 \mathrm{~g} / \mathrm{L}$ Coomassie Brilliant Blue and destained in 10\% Acetic Acid, 25\% Ethanol solution.

\section{CheA CheW Crosslinking}

Samples of $10 \mu \mathrm{M}$ CheA with and without $10 \mu \mathrm{M}$ CheW were incubated in $50 \mathrm{mM}$ HEPES pH 8.0, $150 \mathrm{mM} \mathrm{KCl}, 0.5 \mathrm{mM}$ EDTA, $10 \mathrm{mM} \mathrm{MgCl}_{2}$ and crosslinked with $12.7 \mathrm{mM}$ DSSO for 40 minutes $^{42}$. The reaction was quenched with $20 \mathrm{mM}$ Tris buffer, and the samples subjected to gel electrophoresis on a $4-20 \%$ Tris-glycine protein gel. The gel was stained in $10 \%$ acetic acid, $25 \%$ ethanol in water containing $2.5 \mathrm{~g} / \mathrm{L}$ Coomassie Brilliant Blue and destained in 10\% acetic acid, $25 \%$ ethanol solution.

\section{Fluorescence-Monitored TNP-ATP Binding}

All experiments were performed in $50 \mathrm{mM}$ Tris $\mathrm{pH} 8.0,150 \mathrm{KCl}, 10 \mathrm{mM} \mathrm{MgCl}_{2}$ and with continual stirring with a magnetic stirbar at room temperature in a quartz cuvette. Aliquots of TNP-ATP were added to a temperature-controlled quartz cuvette containing $1 \mathrm{~mL}$ of CheA ( $1 \mu \mathrm{M}$ monomer, $0.5 \mu \mathrm{M}$ dimer). Binding isotherms at $20^{\circ} \mathrm{C}$ were constructed using integrated fluorescence between 500 and $650 \mathrm{~nm}$ following excitation at $410 \mathrm{~nm}$. Integration was performed using the trapezoid rule (average of left and right Riemann sums). Effective $I_{50}$ TNP-ATP values were calculated using MATLAB by fitting the isotherm to the following equation, where $f_{T N P-A T P}($ bound) indicates fractional fluorescence:

$$
f_{T N P-A T P}(\text { bound })=1-\left(\frac{a}{1+e^{b\left(\log _{10}[A D P]-\log _{10}\left[I C_{50}\right]\right)}}+c\right)
$$

To calculate effective $I_{50}$ values for ADP, CheA variants and TNP-ATP were added to solution in equimolar amounts $(5 \mu \mathrm{M})$ in a $1 \mathrm{~cm} \times 1 \mathrm{~cm}$ fluorescence cuvette and binding was monitored by 
fluorescence emission at $550 \mathrm{~nm}$ with an excitation wavelength of $410 \mathrm{~nm}^{43}$. ADP was added from a stock concentration of $1 \mathrm{M}$. Effective $\mathrm{IC}_{50}$ ADP values were calculated using MATLAB:

$$
f_{T N P-A T P}(\text { bound })=\frac{a}{1+e^{b\left(\log _{10}[T N P-A T P]-\log _{10}\left[I C_{50}\right]\right)}}+c
$$

\section{TNP-ATP / ADP Chase Experiment}

CheA WT $(5 \mu \mathrm{M})$ and TNP-ATP $(5 \mu \mathrm{M})$ were incubated at room temperature and stirred continuously with a magnetic stir bar. The sample was excited with $410 \mathrm{~nm}$ light and fluorescence emission at 550 $\mathrm{nm}$ was monitored. $5 \mu \mathrm{L}$ of $1 \mathrm{M} \mathrm{ADP}$ was added and the fluorescence decay monitored.

Fluorescence decay was fit to an exponential decay function using MATLAB. CheA V350C was used out of convenience. CheA V350C does not differ from CheA WT in its nucleotide binding properties or activity profile.

\section{Size-Exclusion Chromatography Small-Angle X-Ray Scattering (SEC-SAXS)}

SAXS data were collected at CHESS G1 line on a Finger Lakes CCD detector. $100 \mu \mathrm{L}$ of CheA WT and CheA-LV1 samples containing 4.5-5.5 mg/mL protein was injected onto a Superdex 200 Increase 10/300 Column pre-equilibrated with sample buffer (50 mM HEPES pH 8.0, 150 mM KCl, 10 mM $\mathrm{MgCl}_{2}$ ) coupled to the G1 SAXS sample cell. The monodisperse stream was fed through a Wyatt MALS/DLS and dRI detector and data was collected with 2 second exposure times to the x-ray beam at a flow rate of roughly $0.2 \mathrm{ml} / \mathrm{min}$. RAW was used to process the SEC-SAXS data and to generate Guinier Fits and dimensionless Kratky Plots ${ }^{44,45}$. FoXS was used to fit the scattering data to a structure of the full-length CheA dimer in its inhibited conformation and compare experimental scattering profiles to the expected scattering profile projected from the model ${ }^{46,47}$. The inhibited conformation of dimeric CheA used was derived from the model constructed by Muok et al, excluding TarFo Short and CheW $^{30}$.

\section{ESR Spectroscopy Measurements}

Proteins were incubated with more than a 500-fold molar excess of ADP-NO at $4{ }^{\circ} \mathrm{C}$ for upwards of 12 hrs. Continuous wave ESR (cwESR) experiments were carried out at X-band ( 9.4 GHz) on a Bruker E500 spectrometer. The cwESR experiments were performed at room temperature with a modulation amplitude of 1-2 G. For 4-pulse-DEER, the samples were exchanged into deuterated buffer containing $30 \% d^{8}$-glycerol, checked by cwESR and plunge frozen into liq. $\mathrm{N}_{2}$. The pulse ESR measurements were carried out at Q-band $(\sim 34 \mathrm{GHz})$ on a Bruker E580 spectrometer equipped with a $10 \mathrm{~W}$ solid state amplifier (150W equivalent TWTA) and an arbitrary waveform generator

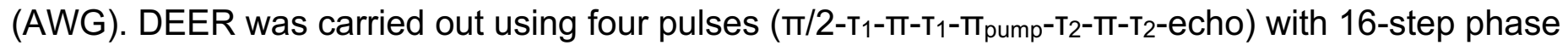


cycling at $60 \mathrm{~K}$. The pump and probe pulses were separated by $56 \mathrm{MHz}(\sim 20 \mathrm{G})$. The DEER data was background subtracted and the distance reconstruction was carried out using denoising and the SVD method ${ }^{48,49}$.

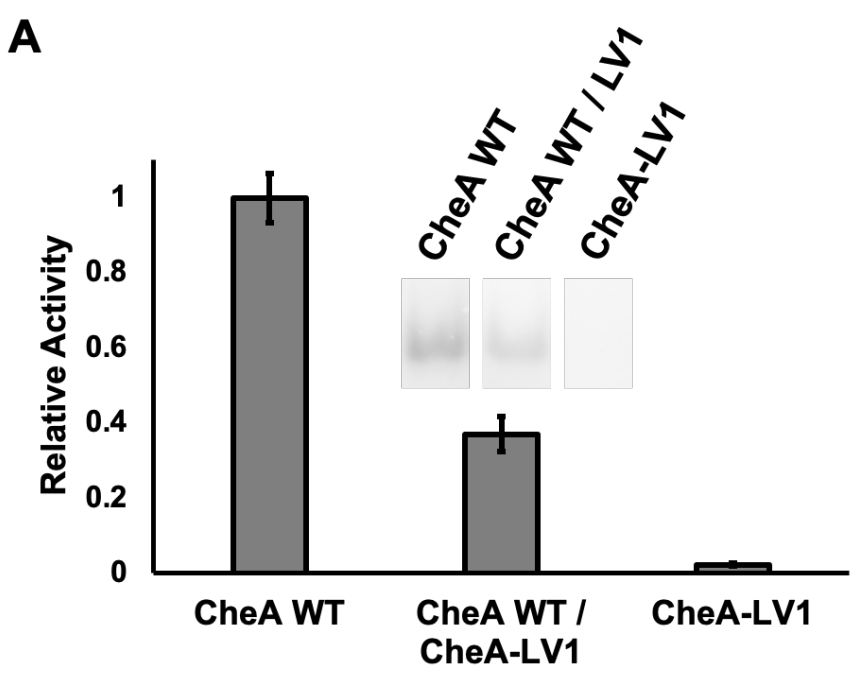

B
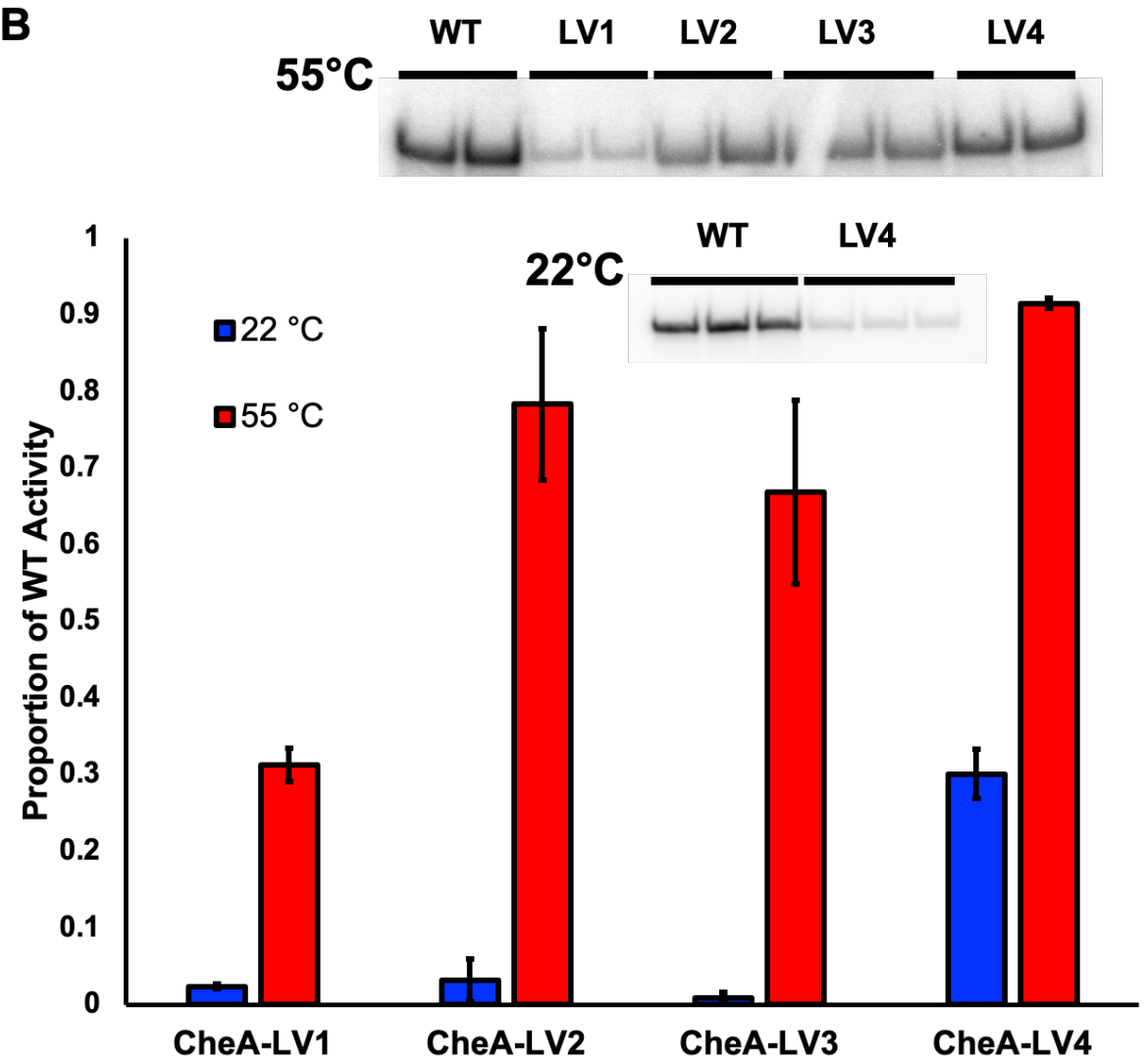

Figure 2 CheA autophosphorylation activity at $22^{\circ} \mathrm{C}$ and $55^{\circ} \mathrm{C}$ (A) Autophosphorylation of CheA WT, CheA-LV1, and heterodimers with mixed subunits. All samples were heated at $55^{\circ} \mathrm{C}$ for 10 minutes; the [P4] is the same among samples. The CheA WT / LV1 sample contains $50 \%$ CheA WT and $50 \%$ CheA-LV1. The inset shows representative bands. (B) Autophosphorylation was measured for each 
variant by radioisotope incorporation from $\mathrm{Y}^{-32} \mathrm{P}-\mathrm{ATP}$ at $22{ }^{\circ} \mathrm{C}$ and $55^{\circ} \mathrm{C} . \quad \mathrm{N}=2$ for all data. Error bars represent mean deviation.

\section{Results}

Preparation, Purification, and Activity of CheA WT and Linker Variants

To probe how the connections between P3 and P4 and P4 and P5 influence kinase activity, residues were inserted into the L3 and L4 linkers or the native residues were substituted for others that favor enhanced flexibility or helical structure (Figure 1C). Specifically, the P4 domain was identified in fulllength Tm CheA as residues 355-538 based on crystal structures of the P3-P4 unit (PDB code: 4XIV) and of the P4-P5 unit (PDB code: $2 \mathrm{CH} 4)^{29,50,51}$. The amino acid sequence of all CheA variants (LV1, $2,3,4)$ are identical to that of CheA WT save for the alterations to L3 and L4. Flexible LV1 was designed to disconnect the P4 domain from structural constraints imposed by P3 and P5. LV2 introduces a helical heptad that could interact across the dimer interface between P3 and P4 and promote an in-register helical connection; LV3 is the same length as LV2 but should be more flexible. LV4 substitutes the specific residues in the WT linker to Ala, including the helix-breaking residue P357, but retains WT spacing. CheA WT and all variants express well in E. coli cells and register at the same molecular weight by SDS-PAGE analysis. Analytical size-exclusion chromatography verified that all proteins are dimeric.

CheA-LV1 autophosphorylation activity was tested against CheA WT by radioisotope incorporation from $\mathrm{Y}^{32}{ }^{32} \mathrm{P}-\mathrm{ATP}$. Though not completely inactive, CheA-LV1 autophosphorylates to only about $2 \%$ that of WT at $22{ }^{\circ} \mathrm{C}$ (Figure 2A). The Tm P4 domain expresses in BL21 cells by itself, is stable, and has been crystalized ${ }^{50}$. Given the stability of the isolated $\mathrm{P} 4$ and the evidence delineated below that misfolding of the P4 domain in CheA-LV1 is unlikely. The autophosphorylation level of CheA-LV1 is similar to the level of kinase activity observed for $P 4$ alone ${ }^{52}$. Extension of $L 3$ and $L 4$ renders the $P 4$ domain in roughly the same proximity to the P1 domain as in CheA WT, and thus, the dramatic impairment of $\mathrm{P} 1$ phosphorylation is not easily explained.

Increased P4-P4 interactions in CheA-LV1, conferred by the flexible linkers, may be responsible for the depression of kinase activity. Effects on WT P4 in a CheA WT / CheA-LV1 heterodimer were investigated. Temperature-driven subunit exchange between $\operatorname{Tm}$ CheA dimers at $55^{\circ} \mathrm{C}$ has been previously used to produce heterodimeric species ${ }^{52}$. Tm CheA WT retains its level of activity after being heated at $55^{\circ} \mathrm{C}$ for 10 minutes and then cooled to room temperature ${ }^{51}$. Assuming equal exchange between equimolar WT and CheA-LV1 subunits, samples containing 1/4 CheA WT 
homodimer, 1/4 CheA-LV1 homodimer, and 1/2 CheA WT / CheA-LV1 heterodimer would be expected (Figure 2A). The roughly 37\% WT activity of the heat exchanged samples containing $50 \%$ WT P4 indicates both that CheA-LV1 kinase activity is not rescued by incorporation into a heterodimer with CheA WT and that a CheA WT subunit in a CheA WT / CheA-LV1 heterodimer also has reduced autophosphorylation activity. Autophosphorylation at less than $50 \%$ activity supports the conclusion that subunit exchange must be occurring between CheA WT and CheA-LV1 and that the LV1 subunit reduces the activity of the WT subunit in a heterodimer.

At $22^{\circ} \mathrm{C}$, CheA-LV2 and CheA-LV3, which extends the L3 linker further by two residues, display very low levels of kinase activity that are comparable to that of CheA-LV1. In contrast, CheA-LV4, which only changes the L3 sequence to poly-Ala has autophosphorylation activity at $22{ }^{\circ} \mathrm{C}$ that is $23-30 \%$ of WT levels. At $55^{\circ} \mathrm{C}$, activity relative to WT increases dramatically for LV2, LV3 and LV4 (Figure 2B). The fact that CheA-LV1 kinase activity at $55^{\circ} \mathrm{C}$ is much less than that of CheA-LV3, which differs from CheA-LV1 only by a Gly-Ser addition, implies that the L3 linker imparts some degree of positioning to $\mathrm{P} 4$ and at $55^{\circ} \mathrm{C}$, and that a helical heptad spacing favors autophosphorylation activity. Furthermore, a sequence designed to stabilize hydrophobic packing of a helical heptad in LV2 produces an even more active conformation than LV3. CheA-LV4 activity is roughly $30 \%$ of WT at 22 ${ }^{\circ} \mathrm{C}$, and all but indistinguishable from WT at $55^{\circ} \mathrm{C}$. CheA-LV4 differs from WT only in the sequence of the L3 element, further evidence of a link between P4 orientation and kinase activity. 
A

\begin{tabular}{l|ll}
\multicolumn{1}{c}{} & \multicolumn{1}{c}{ Effective IC $_{50 \text { TNP-ATP }}$} & Effective IC ${ }_{50}$ ADP \\
\cline { 2 - 3 } CheA WT & $0.28 \pm 0.04 \mu \mathrm{M}$ & $405 \pm 20 \mu \mathrm{M}$ \\
CheA-LV1 & $0.4 \pm 0.2 \mu \mathrm{M}$ & $700 \pm 300 \mu \mathrm{M}$
\end{tabular}

\section{C}

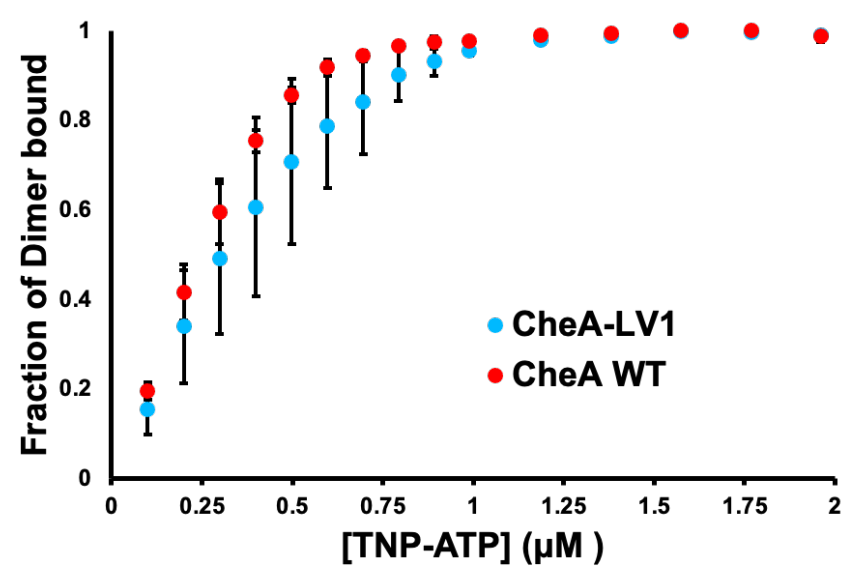

B

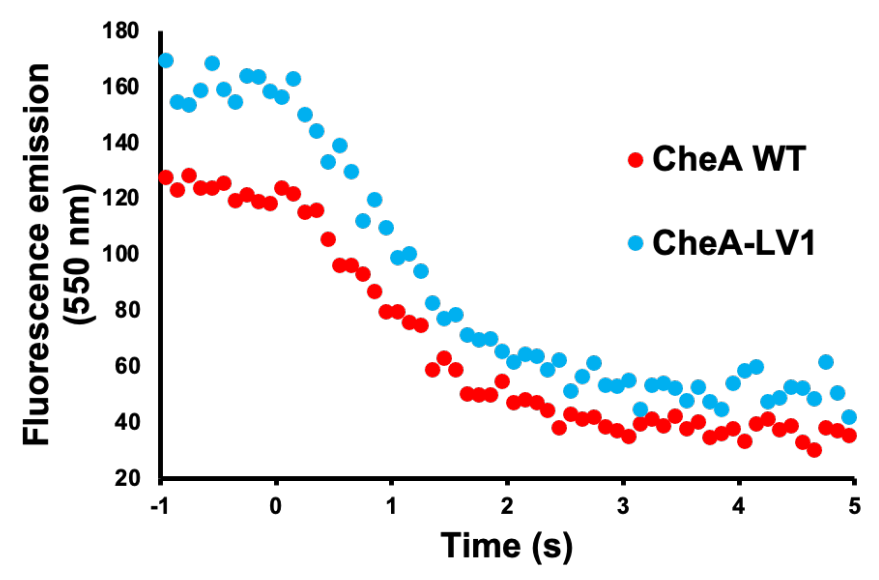

D

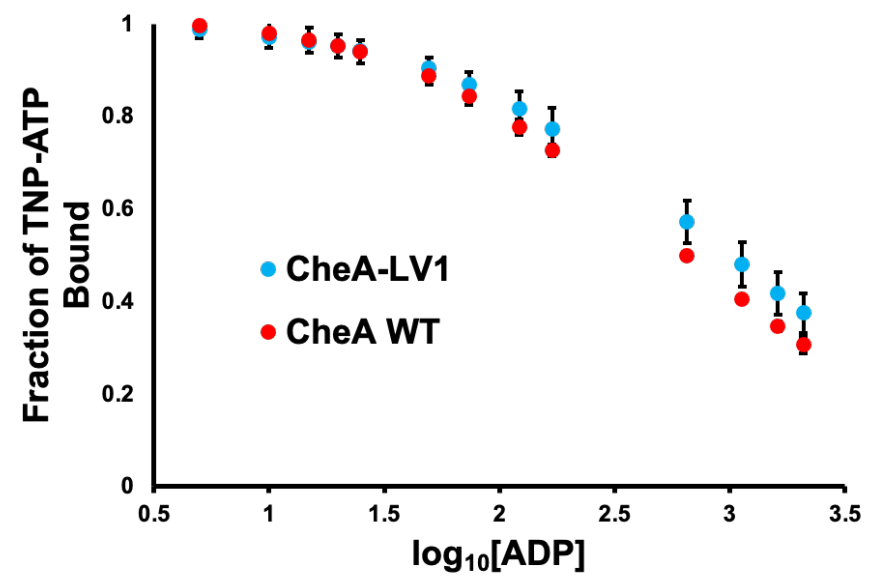

Figure 3 CheA-LV1 binds nucleotide similarly to CheA WT. $N=2$ for all measurements. (A) Table summarizing the nucleotide binding data measured for CheA WT and CheA-LV1. (B) ADP exchange experiment. Addition of 1000-fold molar excess of ADP to CheA WT and CheA-LV1 bound to equimolar TNP-ATP results in nucleotide exchange. Fitting of the decay curves to a first order function gave TNP-ATP $k_{\text {off }}$ rates of $0.45 \pm 0.10$ and $0.40 \pm 0.131 / \mathrm{sec}$ for CheA WT and CheA-LV1, respectively $(\mathrm{N}=2)$. Measurement of $K_{o n}^{T N P-A T P}$ rates was precluded by fast binding of TNP-ATP to P4. (C) TNP-ATP binding to CheA WT and CheA-LV1. Stepwise addition of TNP-ATP to CheA WT and CheA-V1 gives effective IC 50 values of $0.28 \pm 0.04$ and $0.4 \pm 0.2 \mu \mathrm{M}$ (D) TNP-ATP exchange with ADP. Stepwise addition and equilibration of ADP to CheA WT and CheA-LV1 that are initially bound to TNP-ATP gives effective $I_{50}$ values for ADP of $400 \pm 20$ and $700 \pm 300 \mu \mathrm{M}$, respectively $(\mathrm{N}=2)$.

\section{Adenosine Nucleotide Binding Affinity}

To evaluate ATP binding of the inactive CheA-LV1, we first compared the nucleotide binding properties of CheA-LV1 to WT with the fluorescent ATP analog TNP-ATP- $\left(\mathrm{Mg}^{2+}\right)$. TNP-ATP- $\left(\mathrm{Mg}^{2+}\right)$ has weak fluorescence free in solution but strong fluorescence upon binding to the CheA P4 
domain ${ }^{24,53}$. Note that TNP-ATP is a poor phosphoryl donor to CheA $24,43,50$, which is well explained by the structure of TNP-ATP-( $\left.\mathrm{Mg}^{2+}\right)$ bound to P4 (PDB code: 1I5D), where the sugar ring conformation of TNP-ATP differs from that of ATP analogs.

As with CheA WT, TNP-ATP fluorescence enhances upon binding to CheA-LV1. TNP-ATP (and also ATP) binds to the two Tm CheA subunits with affinities that differ by 3-4-orders of magnitude ${ }^{39}$. At the concentrations of TNP-ATP and protein used, primarily one P4 domain of a CheA dimer is expected to be occupied but some effect of the second site is also expected ${ }^{24,43}$. As our goal was to establish whether nucleotide binding by CheA-LV1 was substantially perturbed relative to WT, effective IC $\mathrm{C}_{50}$ values were calculated to simplify the analysis. Binding isotherms of CheA WT and CheA-LV1 reveal similar $I_{50}$ values for TNP-ATP-( $\mathrm{Mg}^{2+}$ ) (Figure 3AC). CheA WT and CheA-LV1 at a subunit concentration of $1 \mu \mathrm{M}$ appear saturated at near $1 \mu \mathrm{M}$ TNP-ATP, consistent with the extremely tight binding expected for the first subunit and similar to that seen previously for $\mathrm{WT}^{43}$. To investigate dissociation rates, CheA WT and CheA-LV1 were incubated with equimolar TNP-ATP, followed by an addition of 1000-fold molar excess of ADP, Fluorescence decay was then monitored to follow TNPATP release from P4 (Figure 3B). Fitting the decay to a single exponential function reveals comparable TNP-ATP off-rates for CheA WT and CheA-LV1. In a related experiment, the concentration of TNP-ATP was held constant and ADP was titrated into solution up to 450-fold molar excess over TNP-ATP (Figure 3D). IC 50 values calculated for ADP based on the exchange over TNP-ATP in CheA WT and CheA-LV1 (405 $\pm 21 \mu \mathrm{M}$ and $713 \pm 308 \mu \mathrm{M}$, respectively) suggest only minor differences in the affinities of ADP for CheA WT and CheA-LV1 P4. Thus, extension of L3 and L4 appear to have little impact on the nucleotide affinity of P4. It follows that the P4 domain of CheALV1 is well folded and has a nucleotide binding pocket that resembles that of the WT kinase. That said, the slightly weaker affinities observed for both TNP-ATP and ADP by CheA-LV1 may represent a minor long-range structural effect of the linkers on nucleotide binding. 
A

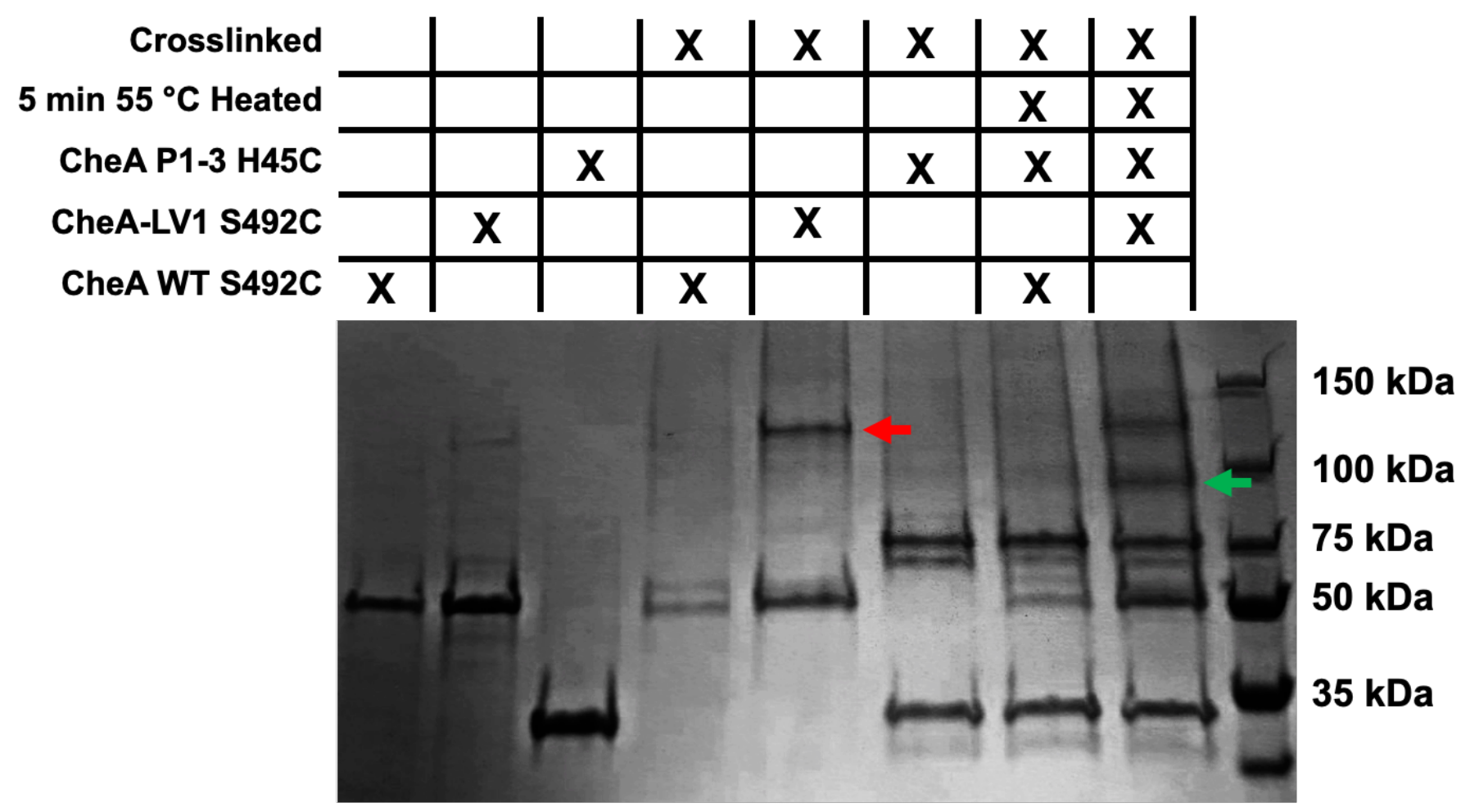

B

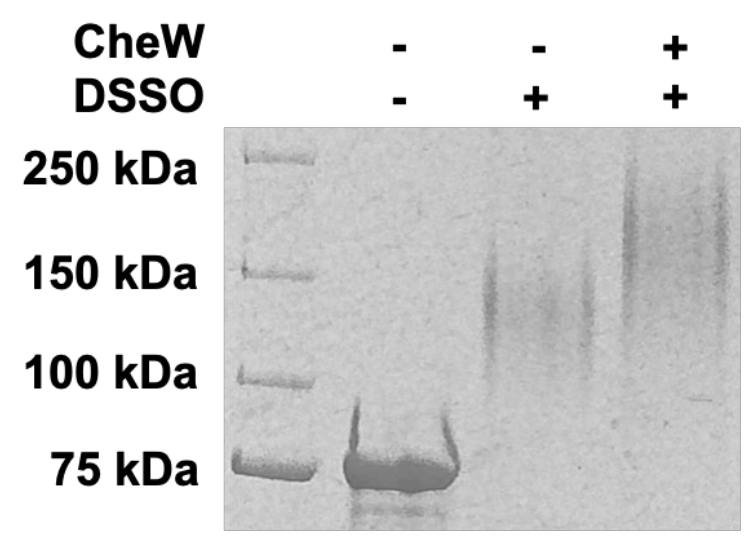

CheA WT

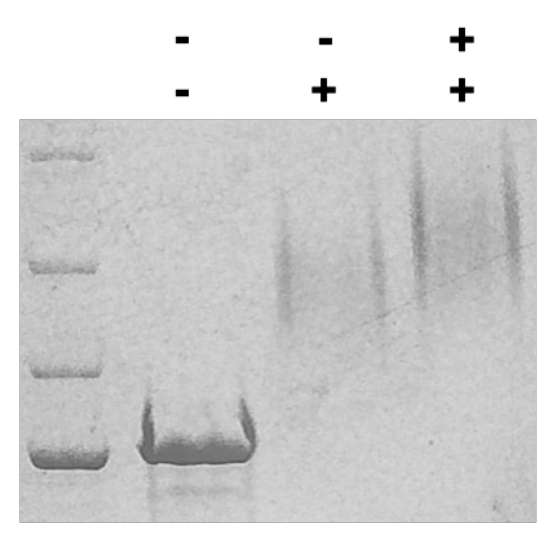

CheA-LV1

Figure 4 Domain and partner interactions of CheA WT and CheA-LV1. (A) Disulfide crosslinking between the P1 domain of CheA P1-3 exchanged and the P4 domain of CheA WT and CheA-LV1 after subunit exchange and addition of $\mathrm{Cu}^{2+}(1,10)$-phenanthroline. Residue 45 on P1 and 492 on P4 were changed to Cys to report on productive substrate kinase interactions. Subunit exchange was provided by heating samples at $55^{\circ} \mathrm{C}$ for 10 mins. In CheA-LV1 crosslinks are found between P4-P4 cross-links (red arrows) and between P1-P4 when exchanged with CheA P1-3 (green arrow). Molecular weight markers shown at right (B) DSSO crosslinking of CheA WT and CheA-LV1 (75 kDa) 
to CheW (17 kDa). Results in $(A)$ and $(B)$ are representative of three equivalent experiments.

\section{Monitoring Inter-domain Interactions with Disulfide Cross-linking}

The Tm CheA subunits do not operate independently. For both Tm and Ec CheA, one subunit of the dimer transphosphorylates the other ${ }^{52,54}$. Furthermore, the negative cooperativity that Tm CheA exhibits upon nucleotide binding indicates that one P4 domain either directly or indirectly influences the other. Previous work has demonstrated that substitutions of S492C in the P4 ATP binding pocket and $\mathrm{H} 45 \mathrm{C}$ of the substrate His residue in the P1 domain cross-link in the presence of $\mathrm{Cu}^{2+}(1,10)-$ phenanthroline (Cu-phen) ${ }^{52}$. Here we similarly investigated heterodimers that contained only one P4 domain. Cu-phen treatment of a CheA fragment composed of only domains P1, P2 and P3 (P1-3) containing the H45C substitution yields a dimer band (Figure 4A). Cu-phen treatment of CheA-LV1 with the single P4 substitution S492C also yields a dimer band, which is absent from the corollary CheA S492C sample, thereby signifying a higher degree of association between the P4 domains in a CheA-LV1 dimer than in WT (Figure 4A). Conveniently, thermostable Tm CheA only exchanges subunits at elevated temperatures $\left(55^{\circ} \mathrm{C}\right)^{55}$. Thus, CheA WT and variant homodimers were heated at $55^{\circ} \mathrm{C}$ for 10 minutes to allow for subunit exchange and heterodimer formation. Equimolar amounts of protein were used in individual crosslinking experiments. Heat exchange at $55^{\circ} \mathrm{C}$ between $\mathrm{CheA}$ S492C and CheA P1-3 H45C followed by Cu-phen disulfide crosslinking and SDS-PAGE analysis indicates that only small amounts of a cross-linked heterodimer product formed. In contrast, heat exchange at $55^{\circ} \mathrm{C}$ between CheA-LV1 S492C and CheAP1-3 H45C followed by Cu-phen crosslinking and SDS-PAGE analysis produced a good yield of cross-linked CheA-LV1 / P1-3 heterodimers (Figure 4A). Enrichment for FL CheA / CheA P1-3 heterodimer by size exclusion chromatography was precluded by the similar molecular weights of the heterodimer and FL CheA and CheA P1-3 homodimers. Increasing the L3 and L4 linker lengths may confer greater flexibility of the P3, P4, and P5 domains that allows for increased subunit exchange between CheA P1-3 and CheALV1 than between CheA P1-3 and WT CheA. In addition, the P3 dimer interface may be stabilized when less constrained by connection to P4. Importantly, the heterodimer exchange experiment shows that the P1 substrate His position is able to access the P4 active site in the LV1 protein when the CheA dimer contains only one P4 domain. However, although the P1 can access the P4 domain of LV1, it cannot be phosphorylated to an appreciable degree (Figure S1). Neither an LV1 nor a WT subunit phosphorylates a P1-3 subunit in a heterodimer (Figure S1). Heterodimer formation was confirmed in these experiments by the reduction of WT CheA phosphorylation after heat exchange with P1-3 (Figure S1). 
To test whether changes to L4 affect the folding of the P5 domain we compared binding of CheW to CheA WT and CheA-LV1. CheW, a paralog of P5, binds through a pseudosymmetric hydrophobic interface to the end of the P5 domain ${ }^{13,32}$. Chemical crosslinking with the lysine-specific reagent DSSO was employed to trap the CheA:CheW complex. CheA WT and CheA-LV1 when crosslinked run at their dimer molecular weights. Upon addition of equimolar CheW, the dimer bands shift $\sim 40$ kDa higher in correspondence to the crosslinked 2 CheA subunits and 2 CheW proteins (Figure 4B). Thus, CheW binds effectively to CheA-LV1 despite the extensions of the L3 and L4 linkers and it follows that the CheA P5 domain is properly folded in CheA-LV1.

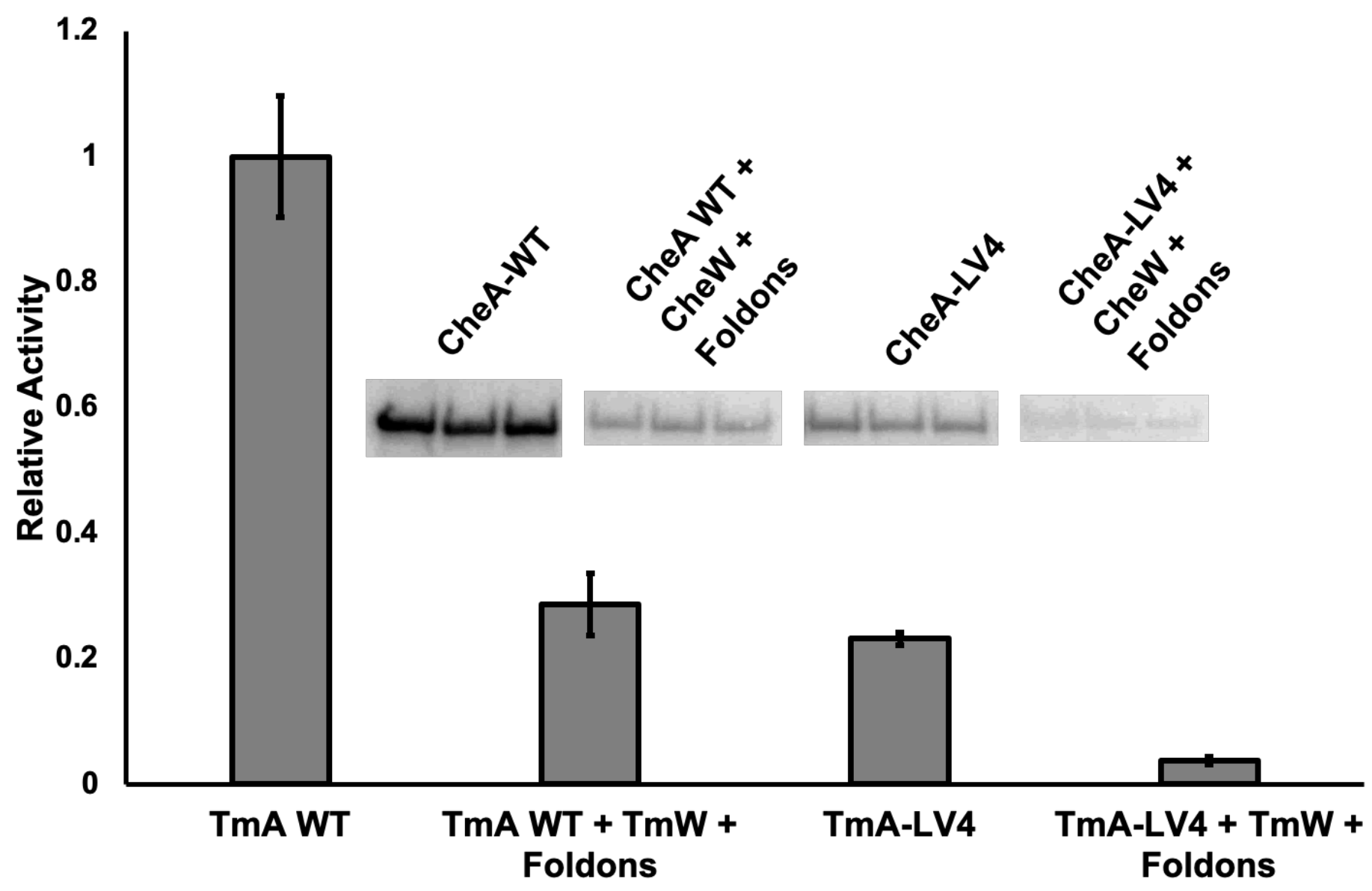

Figure 5 Trimerized receptor fragments deactivate CheA-LV4. Autophosphorylation activity, as measured by radioisotope incorporation from Y-32P-ATP, of CheA WT and CheA-LV4 in the absence and presence of CheW and E. coli TarFo Short. As previously demonstrated, Tm CheA activity is reduced in the presence of receptor TOD units. CheA-LV4 also shows reduced in activity, in roughly the same proportion as WT. Insets show the relevant bands corresponding to phosphorylated CheA $(\mathrm{N}=3)$.

\section{CheA-LV4 Activity Regulation in Ternary Complexes}

Soluble, trimeric chemoreceptor mimetics engineered from the kinase control tip region of the $E$. coli aspartate receptor Tar (Tar Foldon Short or TarFo Short) have been used to reproduce the 
interactions between a trimer of chemoreceptor dimers and the Tm CheA dimer in vitro ${ }^{28,30}$. As CheA-LV4 displays appreciable kinase activity at $22^{\circ} \mathrm{C}$ and only differs from CheA WT in sequence of the L3 linker, regulation by Tar Foldons should be detectable.

Autophosphorylation activity for free CheA-LV4 and in the presence of Tar Fo Short and CheW was compared to the activities of CheA WT, also free and in complex (Figure 5). Under these conditions, the Tar Foldons and CheW depress CheA WT activity to $28 \%$ of the free kinase, whereas they depress CheA-LV4 activity to $16 \%$. Comparable depression of kinase activity signifies an intact regulatory mechanism common to both the WT kinase and CheA-LV4. Unfortunately, owing to instability of the receptor mimetics at elevated temperatures, regulation of the other LV variants could not be tested at conditions where they are sufficiently active. 
A

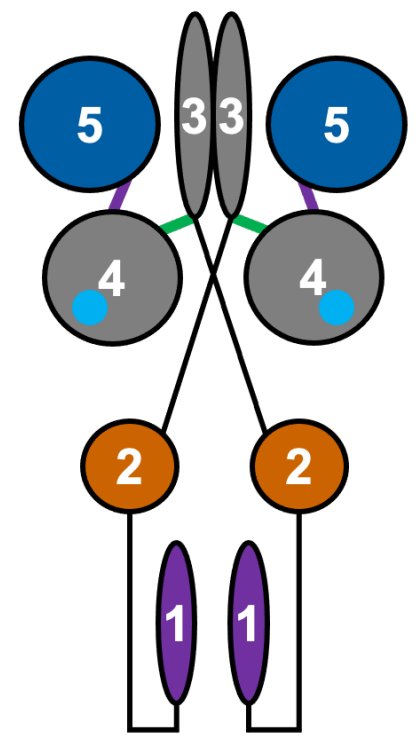

B
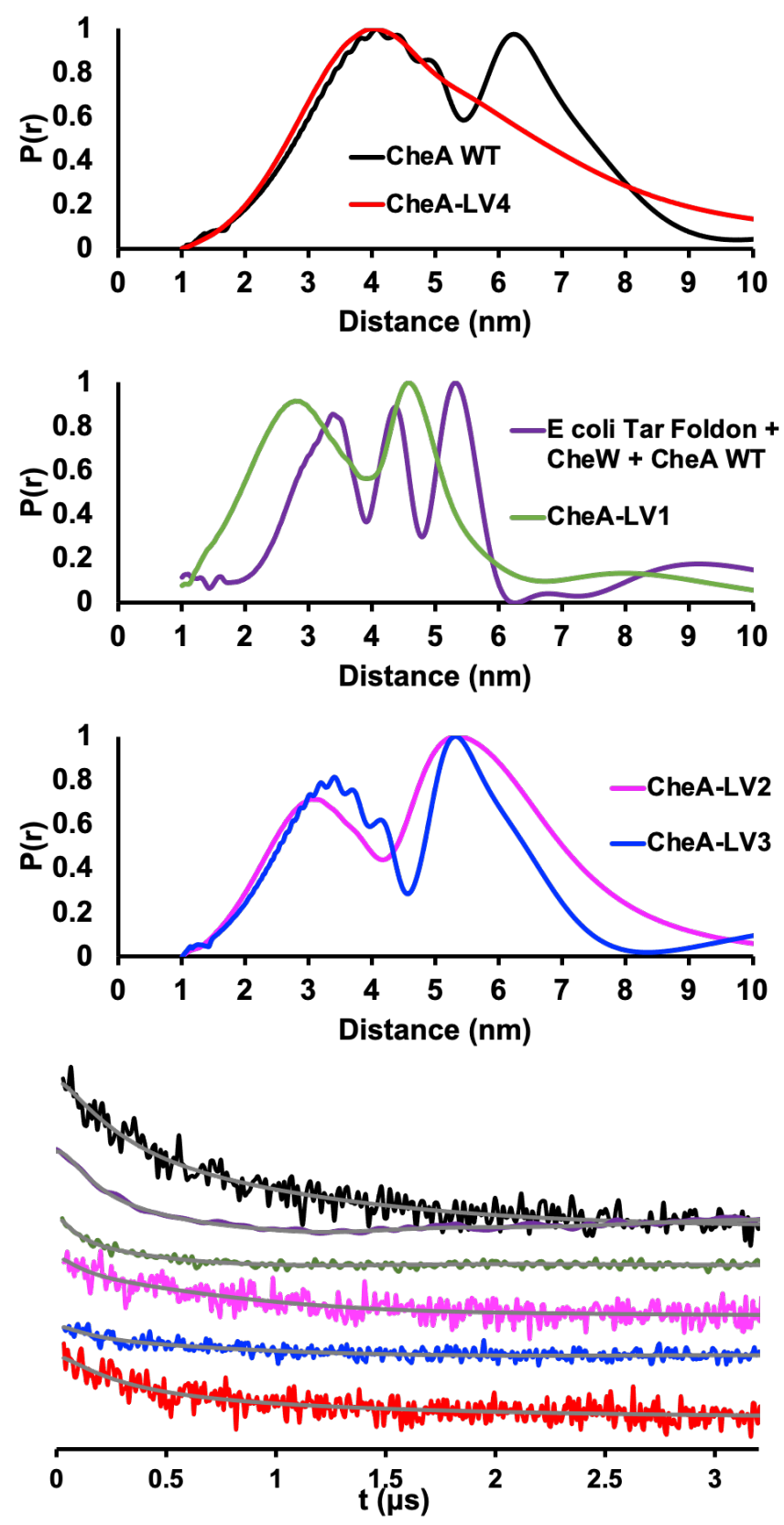

Figure 6 PDS of CheA variants bound to ADP-NO. (A) Cartoon of the CheA dimer showing labeling sites (P1:purple, P2:brown, P34:gray, P5:blue. ADP-NO: cyan circles). (B) DEER-derived distance distributions of ADP-NO bound to CheA WT, linker variants, and foldon-inhibited CheA. Shown below the distance distributions are the time-domain data of the dipolar evolution. PDS data for E. coli Tar Foldon $+\mathrm{CheW}+\mathrm{CheA}$ adapted from Muok and et $\mathrm{al}^{30}$.

\section{P4 Domain Proximity Correlates with Kinase Activity}

The nitroxide modified nucleotide ADP-NO was employed to report on the P4-P4 intradimer distance and P4 dynamics in CheA WT and linker variants using continuous wave and pulse dipolar ESR

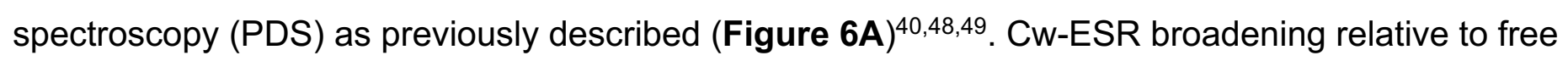


spin-label indicated binding of ADP-NO to the respective P4 domains of each CheA dimer (Figure S2). Low modulation depths in the double electron electron resonance (DEER) time domain data for both CheA-LV1 and CheA WT upon incubation with ADP-NO indicate that ADP-NO does not bind tightly to both subunits, consistent with negative cooperativity in nucleotide binding between the P4 domains $^{39}$. Nonetheless, the DEER data reveals that despite being distributed into a broad set of conformations, the ATP binding sites of the CheA-LV1 dimer are in closer proximity than in CheA WT and resemble the nitroxide separation in the foldon-inhibited CheA WT state (Figure 6B) ${ }^{30}$. This correlation suggests that the CheA-LV1 dimer reflects the domain arrangements of CheA when inhibited by receptors. That said, it is notable that some P4-P4 separations reported by ADP-NO in CheA-LV1 appear even closer than they are in the inhibited receptor complex. Models of the foldon complex sandwich the P1 domains between the distal regions of the P4 domains and thus, the decreased P4-P4 separations may reflect that the P1 domains do not interpose the P4 domains in the inhibited state of CheA-LV130. The SAXS data presented below are consistent with this view.

In contrast, CheA-LV4, the only linker variant with demonstrable autophosphorylation at $22^{\circ} \mathrm{C}$, mimics more closely the long and broad WT separation. That said LV4 shows reduced population of separation at long distances, perhaps suggesting a more restrained conformational landscape. The P4-P4 separations of LV2 and LV3 are similar to each other and intermediate to those of LV1 and LV4. LV2 and LV3 have some short components, but generally shift to longer separations than LV1. Recall that LV2 and LV3 show greatly enhanced activity at elevated temperature, whereas LV1 remains inhibited. Thus, the set of linker variants have P4-P4 separations that generally correlate with their activity state, with shorter distances being indicative of less autophosphorylation activity. 


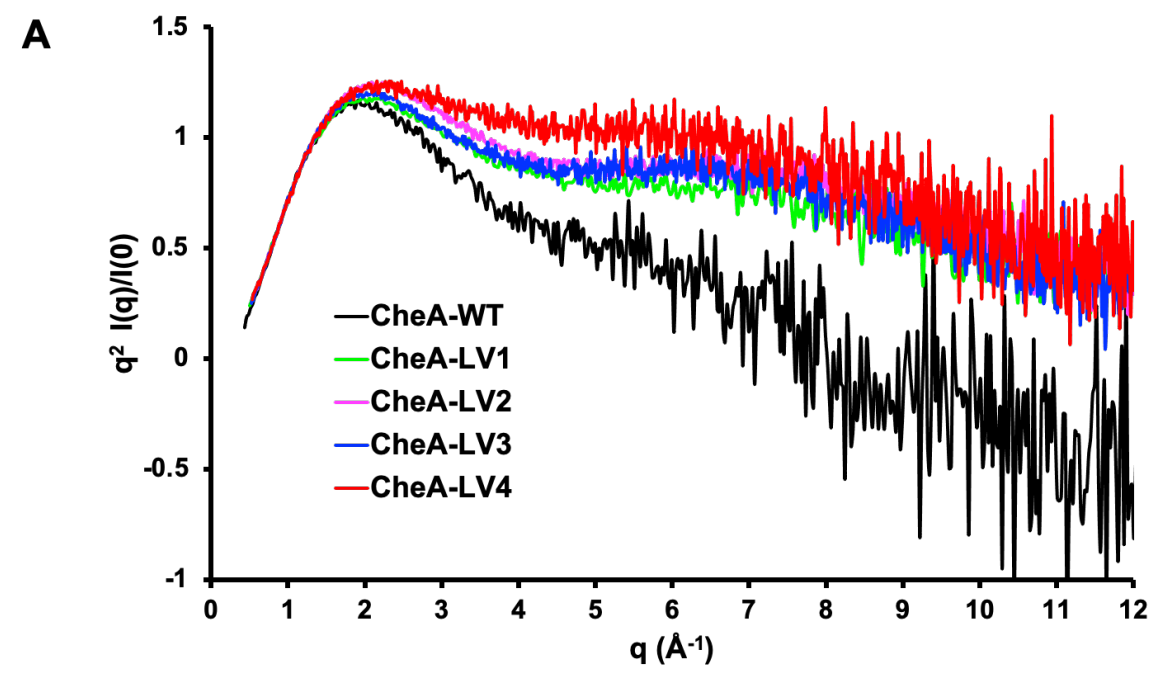

B
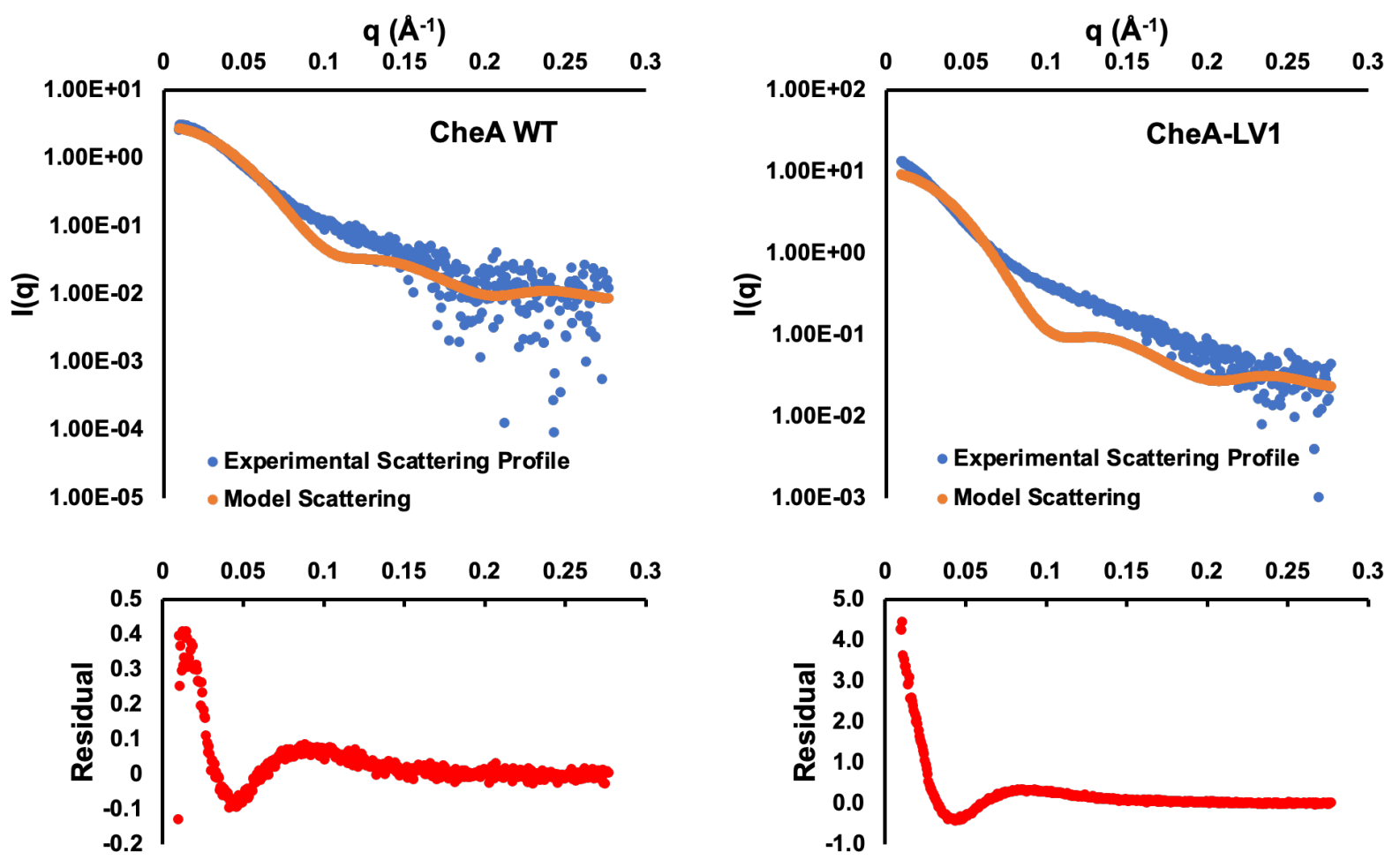

Figure 7 SAXS data on CheA linker variants. (A) Dimensionless Kratky plots of CheA WT and variants showing the mid-q region indicative of flexibility differences ${ }^{52}$. (B) Fits of experimental scattering profiles to a rigid model of inhibited CheA adapted from Muok and et al ${ }^{30}$. Below each fit are residuals comparing the experimentally derived and expected scattering profiles.

Flexibility Analysis by SAXS

The SAXS scattering profiles of CheA WT and the linker variants indicate differences in the conformational properties of the proteins. Dimensionless Kratky plots $\left(q^{2} I[q] v s q\right)$ reveal that all of the variants are substantially less compact than WT (Figure 7A). For the LV proteins, the shift of the Kratky peaks to higher q values and shallower decays in the mid-q region compared to CheA WT indicate less compact states ${ }^{56-59}$. 
CheA-LV1, CheA-LV2, and CheA-LV3 all display comparable scattering profiles, of which CheA-LV1 was used as a prototype. Fitting the scattering profiles for CheA WT and CheA-LV1 to a rigid model of the kinase in its closed, inhibited state corroborates that the scattering profile of CheA WT agrees more closely with the expected scatering profile of the rigid model than does that for CheA-LV1 ${ }^{47}$. The differences between the experimental CheA-LV1 scattering profile and the expected fit for the model suggest that increased P4 dynamics alone cannot account for the change in dynamics in CheA-LV1 (Figure 7B). In particular, the PDS data discussed above show that the P4 domains are more tightly associated in CheA-LV1 than in CheA WT and hence do not likely contribute substantially to the larger hydrodynamic radius. In contrast, we have found that similar changes in protein flexibility, as monitored by SAXS, result from the increased conformational sampling of the P1, $\mathrm{P} 2$ and linker regions as they are released from the core kinase ${ }^{52}$. Thus, it appears that large scale changes in CheA flexibility, likely associated with motion of P1, P2 and possibly L1 and L2 depend on the dynamics and interactions of the P4 domain. This finding is underscored by the behavior of CheALV4. CheA-LV4 has a much less compact scattering profile than CheA WT and all other linker variants, yet CheA-LV4 differs from CheA WT only in the L3 linker sequence, and in neither the L3 linker length nor the L4 linker identity. It is quite remarkable that the large scale changes in the conformational properties of LV4 relative to derive from relatively modest substitutions in L3.

\section{Discussion}

This study corroborates that alterations to the Tm CheA L3 and L4 linker sequences have dramatic effects on kinase activity ${ }^{31,36,37}$. Key questions these data raise include: 1) how are these changes in activity explained in terms of domain structure, interactions and dynamics? and 2) are the behaviors of the variants relevant to the regulation of the native kinase? Previous work has indicated that residue substitutions in L3 reduce both basal autophosphorylation and kinase activation by receptors, whereas changes to L4 can both decrease and increase basal autophosphorylation while reducing kinase activation ${ }^{36,37}$. Two mechanisms of L3 influence on kinase activity of P4 are possible: Firstly, changes to the conformations of L3 and L4 linkers could affect the properties of the P4 kinase machinery. The L3 linker produces differences in structure and dynamics within the P4 active site at residues known to be essential for kinase activity such that L3 modulates P4 catalytic efficiency ${ }^{31}$. In support of this idea, single residue substitutions to L3 (M322A, R325A, M326A) decrease kinase activity $^{37}$. Proper regulation of kinase activity requires signal conveyance from the CheW / P5 layer to P4 through L4 and single residue substitutions in L4 (L507A, T508A, L509A) do not support chemotaxis in vivo ${ }^{37}$. Additionally, substitution of only four residues in L4 in a variant devoid of the P5 domain increase and decrease kinase activity relative to CheA $\triangle \mathrm{P} 5$ and apparently shift $\mathrm{CheA}$ 
towards active and inactive conformations ${ }^{36}$. Secondly, a wider range of $\mathrm{P} 4$ domain orientations are accessible by altering the conformations of L3 and L4 and linker dynamics then gate P4 access to $\mathrm{P} 1^{37}$. To investigate the relevance of these two possibilities for the CheA catalytic cycle, four variants of the L3 and L4 elements were constructed and characterized. These results support the conclusion that the inhibited state of CheA is characterized by close association of the P4 domains, but indirect effects of the linkers on the properties of the ATP binding pocket and the dynamics of the P1 substrate domain are also at play, as evidenced by the absence of CheA P1-3 phosphorylation in CheA WT / CheA P1-3 and CheA-LV1 / CheA P1-3 heterodimers.

Inactivity of CheA-LV1 results from neither impairment to ATP binding, misfolding of P4 nor misfolding of $P 5$

$A$ CheA variant, CheA-LV1, was altered to detach the P4 domain from regulation through altering the conformation and dynamics of its interdomain linkers with P3 and P5. Within a CheA-LV1 dimer, the local concentration of the P4, P2, and P1 domains remain the same as in WT CheA. Compared to CheA WT, CheA-LV1 autophosphorylation activity is reduced 50 -fold. Thus, the residue differences between CheA WT and CheA-LV1 disrupt some critical set of interactions necessary for WT CheA function or serve to trap CheA in a state that prevents the normal catalytic cycle. Despite defects in catalytic activity, the P4 domain of CheA-LV1 retains nucleotide affinity and dissociation rates comparable to CheA WT and the ability to bind CheW. The comparable nucleotide binding affinities of CheA WT and CheA-LV1 implies that both P4 domains are well-folded and that the closer association of the $\mathrm{P} 4$ domains does not prevent nucleotide exchange. The retention of CheW binding affinity to CheA-LV1 indicates that the P5 domain also assumes the expected structure.

\section{CheA-LV1 P4-P4 proximity}

CheA WT / CheA-LV1 heterodimer formation through temperature-driven subunit exchange does not rescue CheA-LV1 catalytic activity and instead results in catalytic impairment of the CheA WT P4 domain. Similarly, negative cooperativity of nucleotide binding in CheA WT is preserved in CheALV1. TNP-ATP binding saturates at a concentration roughly equal to the subunit concentration of CheA. If the P4-P4 communication mechanisms responsible for negative cooperativity in nucleotide binding were substantially perturbed in CheA-LV1, TNP-ATP would not bind a single subunit at the same high affinity as in WT CheA. (Binding in CheA-LV1 would occur at the concentration of CheA monomer, twice the concentration of CheA dimer.) Further evidence in support of this conclusion are the low modulation depths of the nucleotide spin-label DEER experiments after $1 \mathrm{hr}$ of incubation with ADP-NO, indicating that the nucleotide was bound to both CheA subunits in only a small portion of the population in each sample ${ }^{39}$. 
Negative cooperativity in nucleotide binding requires propagation of conformational signals between the P4 domains. As the lengths and sequences of the P4 connections are perturbed in CheA-LV1 by semiflexible GSG-type linkers ${ }^{60}$, L3 and L4 are unlikely to be competent in propagating these signals. If the conformations of the L3 and L4 linkers play a direct role in modulating the nucleotide affinity of P4, more dramatic effects on binding affinity would likely follow from the L3 and L4 variations. Additionally, the close proximity of the P4 ATP binding pockets in CheA-LV1, as evidenced by the PDS data and the S492C cross-linking experiments, indicate a close association of the P4 domains. In particular, the P4-P4 DEER-derived distance distributions show a comparable separation between the ADP binding pocket and rigidity in CheA-LV1 as seen in Ec Tar Fo Short -inhibited CheA. Similar distance distributions between the P4 domains in these two cases corroborate the inactive P4-dimer hypothesis. The striking differences in the P4-P4 distances in CheA WT and CheA-LV1 suggest that upon binding ATP, a P4 domain may inhibit ATP binding of the other P4 domain through either direct allostery or through sequestering the ATP binding site. The observed dimer band upon cysteinecysteine crosslinking of dimeric CheA-LV1 S492C supports the supposition that the ATP binding pockets of the CheA-LV1 P4 domains face one another and thereby block the ATP binding pockets. Furthermore, a decreased distance between the P4 domains of a CheA-LV1 dimer coupled with the observation of negatively cooperative nucleotide binding in both CheA WT and CheA-LV1 suggests that P4-P4 proximity may be contribute to the CheA-LV1 catalytic defect. Given the variability in P4P4 distances, it is possible that changes to L3 and L4 alter the structure of the sequestered state. PDS utilizing ADP-NO bound to CheA WT and linker variants further reveals that kinase activity maximizes when the P4 domains can assume an optimum average distance of separation.

Cooperation between the P4 domains may impact both kinase activation and inhibition. The absence of CheAP1-3 phosphorylation upon heterodimer formation with either FL CheA-LV1 (Figure S1A) or FL CheA WT (Figure S1B), despite direct evidence of interaction between the CheA-LV1 P4 ATP binding site and the CheA P1-3 P1 domain, suggests that each P4 domain is important for regulating the activity of the opposing subunit. Such cooperation may be why a P4 domain has low activity when isolated, but much higher activity when fused to the P3 domains ${ }^{52}$. The absence of CheA P1-3 phosphorylation in CheA WT / P1-3 heterodimers suggests that autophosphorylation depends more on having two P4 domains present than the influence of the P3 domain on one P4 domain. However, it is also possible that a perturbed P3 dimer formed in CheA WT / P1-3 cannot exert the same effect on the single P4 domain. Regardless, only one P4 domain is present in a CheA-LV1 / CheAP1-3 heterodimer and its lack of activity, therefore, cannot result from P4-P4 interactions preventing access of $\mathrm{P} 1$ to the ATP binding site of the CheA-LV1 subunit. Depression of kinase activity in the 
heterodimers may be due in part to P1-P1 dimerization, which is evidenced by CheA P1-3 H45C crosslinking, and by the fact that phosphorylation increases in dimers containing only one P1 domain 52,61,62. Prevention of non-productive $\mathrm{P} 1$ dimerization in the activated state may also require two P4 domains. Likewise, productive interactions of a P1 domain with its adjacent P4 domain may be assisted by its own P4 domain. Although the inability of a WT CheA subunit to phosphorylate a P1-3 subunit suggests that linker-to-active site propagation contributes to the CheA-LV1 catalytic defect, decreased autophosphorylation activity of CheA WT in CheA WT / CheA-LV1 heterodimers strongly indicates that the LV1 P4 directly inhibits the kinase activity of the WT P4.

\section{L3 linker sequence identity and P4 domain motion}

P4 domain positioning and orientation are critical to the $\operatorname{Tm}$ CheA catalytic cycle ${ }^{4,30,38}$. The increased autophosphorylation activity of CheA-LV3 relative to CheA-LV1 at $55^{\circ} \mathrm{C}$ substantiates the hypothesis that L3 helicity is critical to kinase activity. CheA-LV3 is longer than CheA-LV1 by two residues and should thereby allow the WT-like orientations of the two P4 domains if those domains are juxtaposed by continuous helices extending from P3; however, the GSG-type sequence should be quite flexible, especially at higher temperatures. It is thus somewhat surprising that two additional residues increase autophosphorylation as much as they do. However, CheA-LV2 has an L3 sequence that should produce a more stable two-turn helix and these substitutions restore activity to a greater degree than seen in LV3. The activity of CheA-LV4, particularly at elevated temperatures, underscores the importance of L3 helicity in the CheA catalytic cycle. CheA-LV4 differs from CheA WT only in seven substituted Ala residues, which likely promote an $\alpha$-helix between the P3 and P4 domains, while removing a conserved helix-breaking Pro residue. The ability of Ec TarFo Short to regulate CheA-LV4 indicates that the conformational transformations imposed on the CheA-LV4 dimer by the receptor foldons, in the presence of $\mathrm{CheW}$, are not perturbed by these substitutions in L3. The secondary structural change in L3 between $\beta$-strand and kinked $\alpha$-helix proposed to be critical for kinase activity may indeed play a role in the transition between P4 conformational states $^{30,38}$.

$P 4$ conformation and dynamics is coupled to release of P1-2

Conformational characterization by SAXS reveals that all linker variants have considerably more conformational flexibility than CheA WT at room temp, although this increase in conformational flexibility appears to be inconsequential to basal kinase activity. The increase in conformational dynamics of CheA-LV1 relative to CheA WT is conspicuous because the P4 domains are more tightly associated in CheA-LV1. Binding of non-hydrolyzable ATP analog aß-methyleneadenosine-5'- 
triphosphate (ADPCP) to T. maritima CheA has been shown to dramatically increase CheA flexibility, the Kratky plot for which increases with positive slope and plateaus at high q-values, resembling that of a disordered Gaussian chain ${ }^{52}$. Such an increase in global conformational flexibility is liable to result from releasing some regions $\mathrm{N}$-terminal to $\mathrm{P} 3$ from constraint in the globular state. In a similar fashion, the Kratky plots for all CheA linker variants, for which CheA-LV1 is the prototype, decay towards baseline but are considerably more flexible than CheA WT. Although not consistent with complete P1-2 release as observed in CheA WT bound to ADPCP, the Kratky plots for the CheA linker variants are consistent with increased global dynamics. Despite CheA-LV1 assuming an inhibited conformation with associated $\mathrm{P} 4$ domains it also shows enhanced overall domain mobility by SAXS. The flexibility increase must result from changes in P1, L1, P2 or L2 conformational sampling. The constrained conformations of P1 through P2 in the inhibited kinase may depend upon a P4 configuration that is destabilized by the linker variations. Similar arguments hold for CheA-LV2 and CheA-LV3, which have strikingly similar SAXS scattering profiles and dimensionless Kratky plots. Interestingly, CheA-LV4 is even more flexible than any of the other linker variants, yet retains appreciable activity at $22^{\circ} \mathrm{C}$. Furthermore, receptor foldons inhibit CheA-LV4, thereby indicating that the dynamic nature of the full-length free protein does not prohibit inhibition by receptors. Thus, changes to L3 and L4, while enhancing P4-P4 interactions also increase the overall flexibility of fulllength CheA; however, these changes in global flexibility do not appear to increase basal kinase activity.

\section{Consolidating Models}

Several factors likely contribute to impairment of CheA-LV1 autophosphorylation activity. The L3 and L4 linkers may control the movement and / or positioning of the P4 domain and therefore its access to the P1 domain. The L3 and L4 linkers could also alter interactions and conformations internal to the P4 domain and therefore affect catalytic activity indirectly. Molecular dynamics simulations based on cryo-electron microscopy data of signaling units implicate a minor but significant secondary structural change from antiparallel $\beta$-sheet to kinked $\alpha$-helix in the L3 linker over the course of the CheA catalytic cycle ${ }^{16,38}$. This $L 3$ conformational transition has been proposed to alter the hydrogen bonding network between highly conserved residues within the P4 domain and thereby influence P4 positioning relative to the other domains of the CheA dimer. Consistent with this model, previous mutational investigations of $\mathrm{P} 4$ activation revealed that the $\mathrm{L} 3$ linker controls basal CheA autophosphorylation. Furthermore, changes to L3 cause long-range rearrangements in and surrounding the $\mathrm{P} 4$ active site that correlate with effects on CheA activation ${ }^{31,37}$. Modeling of the inhibited kinase:receptor complex of $T m$ CheA bound to Tar $F_{F}$ Short based on PDS measurements, cross-linking, SAXS data and computational optimization portray an inhibited form in which the P4 
domains are closely associated, the P1 and P2 domains are sequestered, and an almost continuous L3 $\alpha$-helix is broken by P35730. In the WT CheA / CheA-LV1 heterodimer, the increased mobility of the CheA-LV1 P4 domain may allow for interactions with the WT P4 domain that prohibit association with P1 or otherwise interrupt the catalytic cycle and thereby depress autophosphorylation of the WT subunit.

The combined data speaks to the importance of P4-P4 interactions for both the inhibition and activation of autophosphorylation and it follows that movements about the P3 linker modulate transitions that alter these interactions and hence enable the catalytic cycle. Temperature activation of the LV proteins to varying degrees indicates a balance between inhibitory interactions, whether they be internal to P4 or between the P4 domains, and domain dynamics that alleviate these contacts. It is quite surprising that the addition of GS in LV3 substantially activates CheA relative to LV1. The fact that this extension recovers a heptad repeat suggests that even these flexible-tosemiflexible GGSG-type linkers ${ }^{60}$ can exert some influence over P4 positioning, by presumably assuming a helix in some stage of the catalytic cycle and splaying the P4 domains away from each other (Figure 8A). Consistent with this notion, a sequence of the same length in LV2, but with greater helix propensity, activates the kinase to a greater extent than LV3. Execution of the catalytic cycle may then involve inhibitory P4-P4 interactions that are relieved by increase in temperature and or by induction of a helical structure in L3 that separates the domains. The P1-3 heterodimer activity experiments suggest that two P4 domains work together to achieve such an active state. This cooperativity may involve direct interactions of the two P4 domains that differ from those of the inhibited state, but could also arise from their coordinated movement, which may be essential for binding and release of one or both of ATP and P125,63.
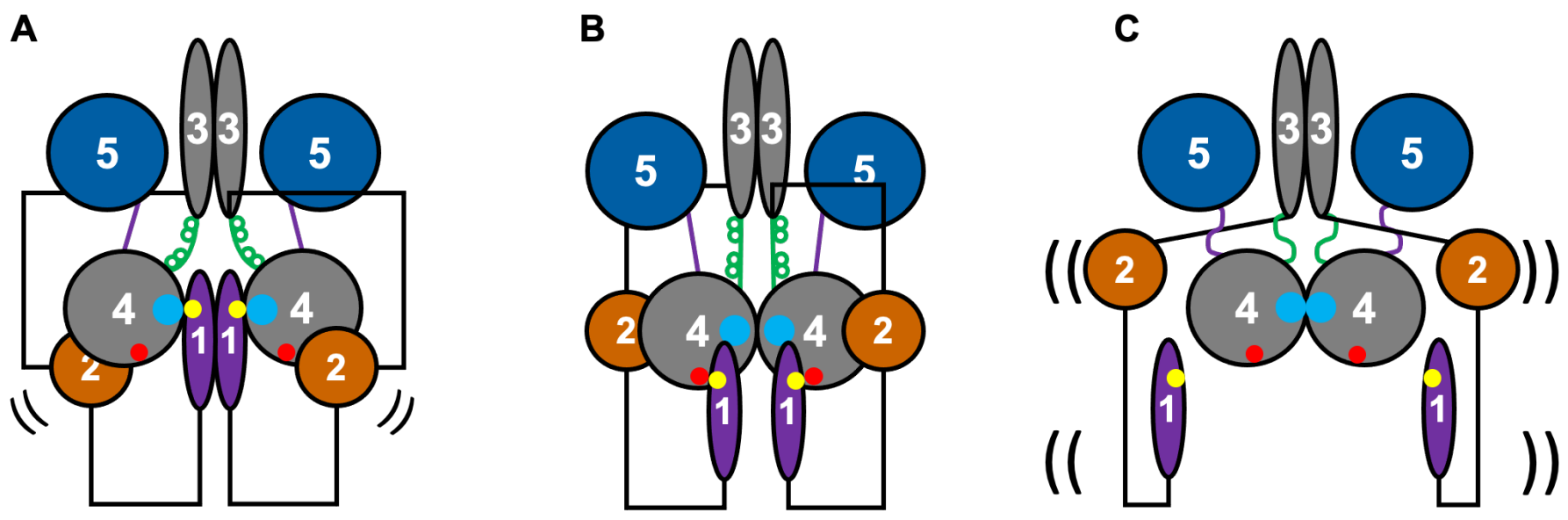

Figure 8 Proposed Linker Effects on P4 Domain Orientation. The L3 linker is shown in green, the L4 linker in purple. The ATP-binding pocket of P4 is shown in cyan, the inhibitory site of P4 in red, and 
the substrate histidine on P1 in yellow. Relative dynamics are indicated. (A) A cartoon representing the domain arrangements of the active free kinase. The L3 linker structure, a broken $\alpha$-helix, plays a role in separating the P4 domains from one another to gate access to the P4 ATP binding site. Note that the associations among P1, P2, and P4 are not as tight as those found in the receptor inhibited complex and may involve a more productive association between P1 and P4 than in the receptorinhibited state in B. (B) A cartoon representing the proposed domain orientations in the receptorinhibited state characterized by an inactive P4-P4 dimer sequestering the P4 ATP binding pockets. P1 binds to a P4 inhibitory site. (C) A cartoon representing the proposed domain orientations adopted by CheA-LV1. The L3 and L4 linkers are extended and flexible. Failure to modulate P4-P4 separation by the extended L3 linker results in an inactive P4 dimer. Occlusion of P1 from P4 results in $\mathrm{P} 1-2$ release and greater flexibility.

Finally, the results also support the notion that the conformational state of P4 influences interactions among P4, P1, and P2 (Figure 8) ${ }^{30}$. Varying degrees of proteolytic protection in different CheA activity states indicates structuring of the $L 1$ and $L 2$ linkers, despite their length and variability across CheA sequences ${ }^{35,64}$ Decoupling of P3 from P4 conferred by lengthening of L3 and L4 causes changes to overall protein flexibility that is too extensive to be explained by P4 movement about the L3 and L4 linkers alone. Hence there must be coupling between P4 mobility and mobility of these otherwise constrained L1 and L2 linkers ${ }^{4,30,34}$. Interactions among P1, P2, and P4, though generating a more compact structure in the free WT protein than in the linker variants, cannot be as tight as in the receptor-inhibited state because free CheA phosphorylates $\mathrm{P} 1$ and does not exhibit chemical cross-linking among $\mathrm{P} 1, \mathrm{P} 2$, and $\mathrm{P} 4$ as does the receptor-inhibited state (Figure $8 \mathrm{AB})^{30}$. The role of such associations is not entirely clear because phosphorylation activity of $\mathrm{E}$. coli CheA can be regulated when P1 is detached from P3-P4 ${ }^{65}$. Nonetheless, there are scenarios where such associations could contribute to regulation in the absence of $\mathrm{P} 1$ attachment ${ }^{4}$. The relative motion of P4 to P3 as mediated by L3 in one subunit may coordinate with motions of the adjacent subunit. The inhibitory interactions, promoted by a lengthened linker, release constraints that poise the P4 domains between active and inactive states (Figure $\mathbf{8 C}$ ). The fact that such constraints can be mitigated by lengthening the linker further to generate a heptad repeat and by increasing temperature suggest that the balance between conformational states that coordinate P1 phosphorylation can be restored and thus these properties are indicative of states normally assumed during CheA catalysis and regulation.

\section{Acknowledgements}


We thank Alise Muok for valuable discussions, Joanne Widom for plasmid construction, and the Cornell High Energy Synchrotron Source (CHESS) and the National Biomedical Center for Advanced ESR Technologies (ACERT) for access to data collection facilities. This work was supported by grants from the National Institutes of Health: R35GM122535 to BRC. ACERT is supported by P41GM103521. CHESS is supported by NSF award DMR-1332208 and NIH/NIGMS award P30GM103485.

\section{References}

(1) Armitage, J. P. Bacterial Tactic Responses. In Advances in Microbial Physiology; Elsevier, 1999; Vol. 41, pp 229-289. https://doi.org/10.1016/S0065-2911(08)60168-X.

(2) Parkinson, J. S.; Hazelbauer, G. L.; Falke, J. J. Signaling and Sensory Adaptation in Escherichia Coli Chemoreceptors: 2015 Update. Trends in Microbiology 2015, 23 (5), 257-266. https://doi.org/10.1016/j.tim.2015.03.003.

(3) Hazelbauer, G. L.; Falke, J. J.; Parkinson, J. S. Bacterial Chemoreceptors: High-Performance Signaling in Networked Arrays. Trends in Biochemical Sciences 2008, 33 (1), 9-19. https://doi.org/10.1016/j.tibs.2007.09.014.

(4) Muok, A. R.; Briegel, A.; Crane, B. R. Regulation of the Chemotaxis Histidine Kinase CheA: A Structural Perspective. Biochimica et Biophysica Acta (BBA) - Biomembranes 2020, 1862 (1), 183030. https://doi.org/10.1016/j.bbamem.2019.183030.

(5) Sultan, S. Z.; Manne, A.; Stewart, P. E.; Bestor, A.; Rosa, P. A.; Charon, N. W.; Motaleb, M. A. Motility Is Crucial for the Infectious Life Cycle of Borrelia Burgdorferi. Infect Immun 2013, 81 (6), 2012-2021. https://doi.org/10.1128/IAI.01228-12.

(6) Rolig, A. S.; Carter, J. E.; Ottemann, K. M. Bacterial Chemotaxis Modulates Host Cell Apoptosis to Establish a T-Helper Cell, Type 17 (Th17)-Dominant Immune Response in Helicobacter Pylori Infection. Proceedings of the National Academy of Sciences 2011, 108 (49), 1974919754. https://doi.org/10.1073/pnas.1104598108.

(7) Howitt, M. R.; Lee, J. Y.; Lertsethtakarn, P.; Vogelmann, R.; Joubert, L.-M.; Ottemann, K. M.; Amieva, M. R. ChePep Controls Helicobacter Pylori Infection of the Gastric Glands and Chemotaxis in the Epsilonproteobacteria. mBio 2011, 2 (4). https://doi.org/10.1128/mBio.0009811.

(8) Kroupitski, Y.; Golberg, D.; Belausov, E.; Pinto, R.; Swartzberg, D.; Granot, D.; Sela, S. Internalization of Salmonella Enterica in Leaves Is Induced by Light and Involves Chemotaxis and Penetration through Open Stomata. AEM 2009, 75 (19), 6076-6086. https://doi.org/10.1128/AEM.01084-09. 
(9) Sze, C. W.; Zhang, K.; Kariu, T.; Pal, U.; Li, C. Borrelia Burgdorferi Needs Chemotaxis To

Establish Infection in Mammals and To Accomplish Its Enzootic Cycle. Infect. Immun. 2012, 80 (7), 2485-2492. https://doi.org/10.1128/IAI.00145-12.

(10) Croxen, M. A.; Sisson, G.; Melano, R.; Hoffman, P. S. The Helicobacter Pylori Chemotaxis Receptor TIpB (HP0103) Is Required for PH Taxis and for Colonization of the Gastric Mucosa. JB 2006, 188 (7), 2656-2665. https://doi.org/10.1128/JB.188.7.2656-2665.2006.

(11) Hazelbauer, G. L.; Lai, W. C. Bacterial Chemoreceptors: Providing Enhanced Features to TwoComponent Signaling. Current Opinion in Microbiology 2010, 13 (2), 124-132.

https://doi.org/10.1016/j.mib.2009.12.014.

(12) Wadhams, G. H.; Armitage, J. P. Making Sense of It All: Bacterial Chemotaxis.

Nat.Rev.Mol.Cell Biol. 2004, 5 (12), 1024-1037. https://doi.org/10.1038/nrm1524.

(13) Briegel, A.; Li, X.; Bilwes, A. M.; Hughes, K. T.; Jensen, G. J.; Crane, B. R. Bacterial Chemoreceptor Arrays Are Hexagonally Packed Trimers of Receptor Dimers Networked by Rings of Kinase and Coupling Proteins. Proceedings of the National Academy of Sciences of the United States of America 2012, 109 (10), 3766-3771.

https://doi.org/10.1073/pnas.1115719109.

(14) Li, M.; Hazelbauer, G. L. Core Unit of Chemotaxis Signaling Complexes. Proceedings of the National Academy of Sciences 2011, 108 (23), 9390-9395.

https://doi.org/10.1073/pnas.1104824108.

(15) Liu, J.; Hu, B.; Morado, D. R.; Jani, S.; Manson, M. D.; Margolin, W. Molecular Architecture of Chemoreceptor Arrays Revealed by Cryoelectron Tomography of Escherichia Coli Minicells.

Proceedings of the National Academy of Sciences 2012, 109 (23), E1481-E1488.

https://doi.org/10.1073/pnas.1200781109.

(16) Cassidy, C. K.; Himes, B. A.; Alvarez, F. J.; Ma, J.; Zhao, G.; Perilla, J. R.; Schulten, K.; Zhang, P. CryoEM and Computer Simulations Reveal a Novel Kinase Conformational Switch in Bacterial Chemotaxis Signaling. eLife 2015, 4, e08419. https://doi.org/10.7554/eLife.08419.

(17) Gloor, S. L.; Falke, J. J. Thermal Domain Motions of CheA Kinase in Solution: Disulfide Trapping Reveals the Motional Constraints Leading to Trans-Autophosphorylation. 14.

(18) Bilwes, A. M.; Alex, L. A.; Crane, B. R.; Simon, M. I. Structure of CheA, a Signal-Transducing Histidine Kinase. 11.

(19) Morrison, T. B.; Parkinson, J. S. Liberation of an Interaction Domain from the Phosphotransfer Region of CheA, a Signaling Kinase of Escherichia Coli. Proceedings of the National Academy of Sciences 1994, 91 (12), 5485-5489. https://doi.org/10.1073/pnas.91.12.5485. 
(20) Stewart, R. C.; Jahreis, K.; Parkinson, J. S. Rapid Phosphotransfer to CheY from a CheA Protein Lacking the CheY-Binding Domain †. Biochemistry 2000, 39 (43), 13157-13165. https://doi.org/10.1021/bi001100k.

(21) Borkovich, K. A.; Kaplan, N.; Hess, J. F.; Simon, M. I. Transmembrane Signal Transduction in Bacterial Chemotaxis Involves Ligand-Dependent Activation of Phosphate Group Transfer. Proceedings of the National Academy of Sciences 1989, 86 (4), 1208-1212. https://doi.org/10.1073/pnas.86.4.1208.

(22) Bourret, R. B.; Borkovich, K. A.; Simon, M. I. Signal Transduction Pathways Involving Protein Phosphorylation in Prokaryotes. Annu. Rev. Biochem. 1991, 60 (1), 401-441. https://doi.org/10.1146/annurev.bi.60.070191.002153.

(23) Levit, M. N.; Liu, Y.; Stock, J. B. Mechanism of CheA Protein Kinase Activation in Receptor Signaling Complexes. Biochemistry 1999, 38 (20), 6651-6658.

https://doi.org/10.1021/bi982839l.

(24) Eaton, A. K.; Stewart, R. C. Kinetics of ATP and TNP-ATP Binding to the Active Site of CheA from Thermotoga Maritima. Biochemistry 2010, 49 (27), 5799-5809. https://doi.org/10.1021/bi100721b.

(25) Jun, S.-Y.; Pan, W.; Hazelbauer, G. L. ATP Binding as a Key Target for Control of the Chemotaxis Kinase. Journal of Bacteriology 2020, 202 (13), 13.

(26) Mello, B. A.; Pan, W.; Hazelbauer, G. L.; Tu, Y. A Dual Regulation Mechanism of Histidine Kinase CheA Identified by Combining Network-Dynamics Modeling and System-Level InputOutput Data. PLoS Comput Biol 2018, 14 (7), e1006305.

https://doi.org/10.1371/journal.pcbi.1006305.

(27) Borkovich, K. A.; Simon, M. I. The Dynamics of Protein Phosphorylation in Bacterial Chemotaxis. Cell 1990, 63 (6), 1339-1348. https://doi.org/10.1016/0092-8674(90)90429-I.

(28) Greenswag, A. R.; Li, X.; Borbat, P. P.; Samanta, D.; Watts, K. J.; Freed, J. H.; Crane, B. R. Preformed Soluble Chemoreceptor Trimers That Mimic Cellular Assembly States and Activate CheA Autophosphorylation. Biochemistry 2015, 54 (22), 3454-3468.

https://doi.org/10.1021/bi501570n.

(29) Greenswag, A. R.; Muok, A.; Li, X.; Crane, B. R. Conformational Transitions That Enable Histidine Kinase Autophosphorylation and Receptor Array Integration. Journal of Molecular Biology 2015, 427 (24), 3890-3907. https://doi.org/10.1016/j.jmb.2015.10.015.

(30) Muok, A. R.; Chua, T. K.; Srivastava, M.; Yang, W.; Maschmann, Z.; Borbat, P. P.; Chong, J.; Zhang, S.; Freed, J. H.; Briegel, A.; Crane, B. R. Engineered Chemotaxis Core Signaling Units Indicate a Constrained Kinase-off State. Sci. Signal. 2020, 13 (657), eabc1328.

https://doi.org/10.1126/scisignal.abc1328. 
(31) Wang, X.; Vallurupalli, P.; Vu, A.; Lee, K.; Sun, S.; Bai, W.-J.; Wu, C.; Zhou, H.; Shea, J.-E.; Kay, L. E.; Dahlquist, F. W. The Linker between the Dimerization and Catalytic Domains of the CheA Histidine Kinase Propagates Changes in Structure and Dynamics That Are Important for Enzymatic Activity. Biochemistry 2014, 53 (5), 855-861. https://doi.org/10.1021/bi4012379.

(32) Piñas, G. E.; DeSantis, M. D.; Parkinson, J. S. Noncritical Signaling Role of a Kinase-Receptor Interaction Surface in the Escherichia Coli Chemosensory Core Complex. Journal of Molecular Biology 2018, 430 (7), 1051-1064. https://doi.org/10.1016/j.jmb.2018.02.004.

(33) Li, X.; Fleetwood, A. D.; Bayas, C.; Bilwes, A. M.; Ortega, D. R.; Falke, J. J.; Zhulin, I. B.; Crane, B. R. The 3.2 A Resolution Structure of a Receptor:CheA:CheW Signaling Complex Defines Overlapping Binding Sites and Key Residue Interactions within Bacterial Chemosensory Arrays. 2013, 14.

(34) Merz, G. E.; Borbat, P. P.; Muok, A. R.; Srivastava, M.; Bunck, D. N.; Freed, J. H.; Crane, B. R. Site-Specific Incorporation of a $\mathrm{Cu}^{2+}$ Spin Label into Proteins for Measuring Distances by Pulsed Dipolar Electron Spin Resonance Spectroscopy. J. Phys. Chem. B 2018, 122 (41), 9443-9451. https://doi.org/10.1021/acs.jpcb.8b05619.

(35) Briegel, A.; Ames, P.; Gumbart, J. C.; Oikonomou, C. M.; Parkinson, J. S.; Jensen, G. J. The Mobility of Two Kinase Domains in the E Scherichia Coli Chemoreceptor Array Varies with Signalling State. Molecular Microbiology 2013, 89 (5), 831-841.

https://doi.org/10.1111/mmi.12309.

(36) Ding, X.; He, Q.; Shen, F.; Dahlquist, F. W.; Wang, X. Regulatory Role of an Interdomain Linker in the Bacterial Chemotaxis Histidine Kinase CheA. J Bacteriol 2018, 200 (10), e00052-18. https://doi.org/10.1128/JB.00052-18.

(37) Wang, X.; Wu, C.; Vu, A.; Shea, J.-E.; Dahlquist, F. W. Computational and Experimental Analyses Reveal the Essential Roles of Interdomain Linkers in the Biological Function of Chemotaxis Histidine Kinase CheA. J. Am. Chem. Soc. 2012, 134 (39), 16107-16110. https://doi.org/10.1021/ja3056694.

(38) Cassidy, C. K.; Himes, B. A.; Sun, D.; Ma, J.; Zhao, G.; Parkinson, J. S.; Stansfeld, P. J.; Luthey-Schulten, Z.; Zhang, P. Structure and Dynamics of the E. Coli Chemotaxis Core Signaling Complex by Cryo-Electron Tomography and Molecular Simulations. Commun Biol 2020, 3 (1), 24. https://doi.org/10.1038/s42003-019-0748-0.

(39) Eaton, A. K.; Stewart, R. C. The Two Active Sites of Thermotoga Maritima CheA Dimers Bind ATP with Dramatically Different Affinities. Biochemistry 2009, 48 (27), 6412-6422. https://doi.org/10.1021/bi900474g. 
(40) Muok, A. R.; Chua, T. K.; Le, H.; Crane, B. R. Nucleotide Spin Labeling for ESR Spectroscopy of ATP-Binding Proteins. Appl Magn Reson 2018, 49 (12), 1385-1395.

https://doi.org/10.1007/s00723-018-1070-6.

(41) Hughson, A. G.; Hazelbauer, G. L. Detecting the Conformational Change of Transmembrane Signaling in a Bacterial Chemoreceptor by Measuring Effects on Disulfide Cross-Linking in Vivo. Proceedings of the National Academy of Sciences 1996, 93 (21), 11546-11551. https://doi.org/10.1073/pnas.93.21.11546.

(42) Kao, A.; Chiu, C.; Vellucci, D.; Yang, Y.; Patel, V. R.; Guan, S.; Randall, A.; Baldi, P.;

Rychnovsky, S. D.; Huang, L. Development of a Novel Cross-Linking Strategy for Fast and Accurate Identification of Cross-Linked Peptides of Protein Complexes. Molecular \& Cellular Proteomics 2011, 10 (1), M110.002170. https://doi.org/10.1074/mcp.M110.002212.

(43) Eaton, A. K.; Stewart, R. C. The Two Active Sites of Thermotoga Maritima CheA Dimers Bind ATP with Dramatically Different Affinities. Biochemistry 2009, 48 (27), 6412-6422. https://doi.org/10.1021/bi900474g.

(44) Hopkins, J. B.; Gillilan, R. E.; Skou, S. BioXTAS RAW: Improvements to a Free Open-Source Program for Small-Angle X-Ray Scattering Data Reduction and Analysis. J Appl Crystallogr 2017, 50 (5), 1545-1553. https://doi.org/10.1107/S1600576717011438.

(45) Franke, D.; Svergun, D. I. DAMMIF , a Program for Rapid Ab-Initio Shape Determination in Small-Angle Scattering. J Appl Crystallogr 2009, 42 (2), 342-346.

https://doi.org/10.1107/S0021889809000338.

(46) Schneidman-Duhovny, D.; Hammel, M.; Tainer, J. A.; Sali, A. Accurate SAXS Profile Computation and Its Assessment by Contrast Variation Experiments. Biophysical Journal 2013, 105 (4), 962-974. https://doi.org/10.1016/j.bpj.2013.07.020.

(47) Schneidman-Duhovny, D.; Hammel, M.; Tainer, J. A.; Sali, A. FoXS, FoXSDock and MultiFoXS: Single-State and Multi-State Structural Modeling of Proteins and Their Complexes Based on SAXS Profiles. Nucleic Acids Res 2016, 44 (W1), W424-W429.

https://doi.org/10.1093/nar/gkw389.

(48) Srivastava, M.; Freed, J. H. Singular Value Decomposition Method to Determine Distance Distributions in Pulsed Dipolar Electron Spin Resonance. Journal of Physical Chemistry Letters 2017, 8 (22), 5648-5655. https://doi.org/10.1021/acs.jpclett.7b02379.

(49) Srivastava, M.; Georgieva, E. R.; Freed, J. H. A New Wavelet Denoising Method for Experimental Time-Domain Signals: Pulsed Dipolar Electron Spin Resonance. Journal of Physical Chemistry A 2017, 121 (12), 2452-2465. https://doi.org/10.1021/acs.jpca.7b00183.

(50) Bilwes, A. M.; Quezada, C. M.; Croal, L. R.; Crane, B. R.; Simon, M. I. Nucleotide Binding by the Histidine Kinase CheA. nature structural biology 2001, 8 (4), 8. 
(51) Park, S.-Y.; Borbat, P. P.; Gonzalez-Bonet, G.; Bhatnagar, J.; Pollard, A. M.; Freed, J. H.; Bilwes, A. M.; Crane, B. R. Reconstruction of the Chemotaxis Receptor-Kinase Assembly. Nat Struct Mol Biol 2006, 13 (5), 400-407. https://doi.org/10.1038/nsmb1085.

(52) Greenswag, A. R.; Muok, A.; Li, X.; Crane, B. R. Conformational Transitions That Enable Histidine Kinase Autophosphorylation and Receptor Array Integration. Journal of Molecular Biology 2015, 427 (24), 3890-3907. https://doi.org/10.1016/j.jmb.2015.10.015.

(53) Stewart, R. C.; VanBruggen, R.; Ellefson, D. D.; Wolfe, A. J. TNP-ATP and TNP-ADP as Probes of the Nucleotide Binding Site of CheA, the Histidine Protein Kinase in the Chemotaxis Signal Transduction Pathway of Escherichia Coli ${ }^{\dagger}$. Biochemistry 1998, 37 (35), 12269-12279. https://doi.org/10.1021/bi980970n.

(54) Swanson, R. V.; Bourret, R. B.; Simon, M. I. Intermolecular Complementation of the Kinase Activity of CheA. Mol Microbiol 1993, 8 (3), 435-441. https://doi.org/10.1111/j.13652958.1993.tb01588.x.

(55) Park, S.-Y.; Quezada, C. M.; Bilwes, A. M.; Crane, B. R. Subunit Exchange by CheA Histidine Kinases from the Mesophile Escherichia Coli and the Thermophile Thermotoga Maritima ${ }^{\dagger}$. Biochemistry 2004, 43 (8), 2228-2240. https://doi.org/10.1021/bi0352419.

(56) Hammel, M. Validation of Macromolecular Flexibility in Solution by Small-Angle X-Ray Scattering (SAXS). Eur Biophys J 2012, 41 (10), 789-799. https://doi.org/10.1007/s00249-0120820-x.

(57) Putnam, C. D.; Hammel, M.; Hura, G. L.; Tainer, J. A. X-Ray Solution Scattering (SAXS) Combined with Crystallography and Computation: Defining Accurate Macromolecular Structures, Conformations and Assemblies in Solution. Quart. Rev. Biophys. 2007, 40 (3), 191285. https://doi.org/10.1017/S0033583507004635.

(58) Rambo, R. P.; Tainer, J. A. Accurate Assessment of Mass, Models and Resolution by SmallAngle Scattering. Nature 2013, 496 (7446), 477-481. https://doi.org/10.1038/nature12070.

(59) Durand, D.; Vivès, C.; Cannella, D.; Pérez, J.; Pebay-Peyroula, E.; Vachette, P.; Fieschi, F. NADPH Oxidase Activator P67phox Behaves in Solution as a Multidomain Protein with SemiFlexible Linkers. Journal of Structural Biology 2010, 169 (1), 45-53.

https://doi.org/10.1016/j.jsb.2009.08.009.

(60) Gräwe, A.; Stein, V. Linker Engineering in the Context of Synthetic Protein Switches and Sensors. Trends in Biotechnology 2021, 39 (7), 731-744. https://doi.org/10.1016/j.tibtech.2020.11.007.

(61) Levit, M.; Liu, Y.; Surette, M.; Stock, J. Active Site Interference and Asymmetric Activation in the Chemotaxis Protein Histidine Kinase CheA. Journal of Biological Chemistry 1996, 271 (50), 32057-32063. https://doi.org/10.1074/jbc.271.50.32057. 
(62) Kott, L.; Braswell, E. H.; Shrout, A. L.; Weis, R. M. Distributed Subunit Interactions in CheA Contribute to Dimer Stability: A Sedimentation Equilibrium Study. Biochimica et Biophysica Acta (BBA) - Proteins and Proteomics 2004, 1696 (1), 131-140.

https://doi.org/10.1016/j.bbapap.2003.10.001.

(63) Piñas, G. E.; Parkinson, J. S. Identification of a Kinase-Active CheA Conformation in Escherichia Coli Chemoreceptor Signaling Complexes. J Bacteriol 2019, 201 (23). https://doi.org/10.1128/JB.00543-19.

(64) Slivka, P. F.; Falke, J. J. Isolated Bacterial Chemosensory Array Possesses Quasi- and Ultrastable Components: Functional Links between Array Stability, Cooperativity, and Order. Biochemistry 2012, 51 (51), 10218-10228. https://doi.org/10.1021/bi301287h.

(65) Pan, W.; Dahlquist, F. W.; Hazelbauer, G. L. Signaling Complexes Control the Chemotaxis Kinase by Altering Its Apparent Rate Constant of Autophosphorylation: Control of the Chemotaxis Kinase. Protein Science 2017, 26 (8), 1535-1546.

https://doi.org/10.1002/pro.3179.

\section{Accession IDs of Proteins from UniProt}

Thermotoga maritima CheY: Q56312

Thermotoga maritima CheW: Q56311

Thermotoga maritima CheA: Q56310 